This item was submitted to Loughborough's Research Repository by the author.

Items in Figshare are protected by copyright, with all rights reserved, unless otherwise indicated.

\title{
Analysis and comparison of wrist splint designs using the finite element method: Multi-material three-dimensional printing compared to typical existing practice with thermoplastics
}

\section{PLEASE CITE THE PUBLISHED VERSION}

https://doi.org/10.1177/0954411917718221

\section{PUBLISHER}

Sage (@ IMechE)

\section{VERSION}

AM (Accepted Manuscript)

\section{PUBLISHER STATEMENT}

This work is made available according to the conditions of the Creative Commons Attribution-NonCommercial 4.0 International (CC BY-NC 4.0) licence. Full details of this licence are available at:

https://creativecommons.org/licenses/by-nc/4.0/

\section{LICENCE}

CC BY-NC 4.0

\section{REPOSITORY RECORD}

Cazon, Aitor, Sarah Kelly, Abby Paterson, Richard Bibb, and lan Campbell. 2017. "Analysis and Comparison of Wrist Splint Designs Using the Finite Element Method: Multi-material Three-dimensional Printing Compared to Typical Existing Practice with Thermoplastics". Loughborough University.

https://hdl.handle.net/2134/25731. 
Original Article

Analysis and comparison of wrist splint designs using the Finite Element Method: multi-material 3D printing compared to typical existing practice with thermoplastics

\section{Authors}

Aitor Cazon ${ }^{1}$, Sarah Kelly², Abby M Paterson², Richard J Bibb² and R. Ian Campbell ${ }^{2}$

${ }^{1}$ Department of Mechanics, Tecnun University of Navarra, Spain

${ }^{2}$ Loughborough Design School, Loughborough University, UK

\section{Corresponding Author}

Abby Paterson. Loughborough Design School, Loughborough University, Loughborough, Leicestershire, LE11 3TU, UK

Email: a.m.paterson@lboro.ac.uk 


\section{Abstract}

Rheumatoid arthritis (RA) is a chronic disease affecting the joints. Treatment can include immobilisation of the affected joint with a custom-fitting splint, which are typically fabricated by hand from Low Temperature Thermoplastic (LTT), but the approach poses several limitations. This study focused on the evaluation, by Finite Element Analysis (FEA), of Additive Manufacturing (AM) techniques for wrist splints in order to improve upon the typical splinting approach. An AM splint, specifically designed to be built using Objet Connex multi-material technology and a virtual model of a typical splint, digitised from a real patient-specific splint using 3D scanning, were modelled in Computer-Aided Design software. Forty FEA simulations were performed in Flexion-Extension and Radial-Ulnar wrist movements to compare the displacements and the stresses. Simulations have shown that for low severity loads, the AM splint has $25 \%, 76 \%$ and $27 \%$ less displacement in the main loading direction than the typical splint in Flexion, Extension and Radial respectively, while Ulnar values were $75 \%$ lower in the traditional splint. For higher severity loads, the Flexion and Extension movements resulted in deflections that were $24 \%$ and $60 \%$ respectively lower in the AM splint. However, for higher severity loading the Radial defection values were very similar in both splints and Ulnar movement deflection was higher in the AM splint. A physical prototype of the AM splint was also manufactured and was tested under normal conditions to validate the FEA data. Results from static tests showed maximum displacements of $3.46 \mathrm{~mm}, 0.97 \mathrm{~mm}, 3.53 \mathrm{~mm}$, and $2.51 \mathrm{~mm}$ Flexion, Extension, Radial and Ulnar directions respectively. According to these results, the present research 
argues that, from a technical point of view, the AM splint design stands at the same or even better level of performance in displacements and stress values in comparison to the typical LTT approach and is therefore a feasible approach to splint design and manufacture.

\section{Keywords}

Arthritis, wrist splint, Additive Manufacturing, Finite Element Analysis, Von Mises Stress

\section{Introduction}

Rheumatoid arthritis (RA) is a chronic disease first described in 1800 by Augustin Jacob Landré-Beauvais that typically affects joints and involves a decline in general health if not properly treated leading to reduced functionality and mobility as well as pain, fatigue and discomfort ${ }^{1}$. Treatment methods may involve patient education, physical therapy and non-steroidal anti-inflammatory medication, but surgery may also be required in extreme cases ${ }^{2,3}$. In addition to treatment methods mentioned previously, Occupational Therapy (OT) is also a common RA treatment that investigates the limitations that patients have in everyday living. By analysing such limitations, Occupational Therapists help patients to develop competence in everyday activities, resulting in a 'maximal functional capacity' ${ }^{2}$ and 'a sense of self-efficacy' ${ }^{4}$. Upper extremity splinting (e.g. static immobilisation wrist splints) is a popular OT 
treatment method that provides immobilisation (by limiting the range of movement of affected joints), protection (a frame to rest the hand/wrist safely without risk of impacts) and symptom relief that enables and encourages patients to carry out everyday activities ${ }^{5}$. Both prefabricated 'off the shelf' and custom-made splints may be prescribed. However, prefabricated splints may not always be appropriate ${ }^{5}$ and subsequently splinting practitioners emphasise customisation of every splint to suit the patient's needs and requirements. This addresses not only fit and functionality but also the patient's psychological wellbeing ${ }^{6}$ in order, for example, to feel aesthetically and emotionally comfortable when wearing the splint in social situations.

Current custom-fitting splints are typically made by hand from sheets of Low Temperature Thermoplastic (LTT) and have several functional and aesthetic limitations. When formed, the typical LTT splint is a solid hard plastic splint. Fasteners such as large buckles and Velcro may be added and are often cumbersome. In terms of aesthetics, patients may have the perceived stigma associated with assistive devices ${ }^{7}$. With regards to function and practicality, patients encounter issues with insufficient skin ventilation ${ }^{8}$ as well as keeping their splints clean and dry ${ }^{9}$ especially in the palmar region which accommodates approximately 500 eccrine sweat glands per square centimetre. Veehof et al. ${ }^{9}$ described induced perspiration for sweat in closed-cell padding, 
whilst Coppard and Lynn ${ }^{10}$ highlighted the issues regarding moisture absorption and collection of perspiration in open-cell padding. The collection of sweat and moisture can result in an unhygienic splint and splints made with traditional methods are currently very difficult to keep clean and dry. Patients may be advised to wash their splints by hand with mild detergents such as washing up liquid, or depending on the materials used, may be advised to avoid washing the splint all together to prevent potential deformation or difficulty with drying the splint. In particular the use of LTT precludes washing at elevated temperatures. Patients may even have two identical splints made, so that one splint may be used for everyday activities which are likely to soil the splint (e.g. gardening, household cleaning), whilst the second splint may be used for other tasks or activities such as cooking or dining. However, the creation of two identical splints is costly in terms of fabrication time, labour and materials. Additionally, poor fit can lead to ulcerations, redness of the skin and pressure sores, particularly on prominent bony landmarks ${ }^{5}$. Unfortunately, these weaknesses cannot be economically circumvented using current fabrication methods, due to restrictions of materials, fabrication methods and clinician time.

Traditional splinting processes are creative and practitioners have to improvise in order to deliver suitable splints that address their patients' needs. Such improvisations and decision-making processes are driven by professional, 
clinical judgement through training and experiential knowledge. Furthermore, fabrication techniques developed by practitioners use tacit knowledge and are therefore difficult to convey to others. Therefore, it is very important for practitioners to remain at the forefront of splint prescription. However, initial findings by Paterson et al. ${ }^{11}$ suggested that practitioners were open to new concepts for splint design and fabrication using three-dimensional (3D) Computer Aided Design (CAD) to support Additive Manufacturing (AM). AM is the process of creating a physical object based on its virtual representation by adding material layer by layer ${ }^{12}$. Its main advantage compared to traditional manufacturing methods is that almost any geometry can be built, offering unlimited and unconstrained geometric complexity: In essence, the statement 'What You See Is What You Build (WYSIWYB)' can often be achieved ${ }^{13}$. This advantage has proved its worth in a number of medical applications, ranging from hearing aids to tissue engineering. Design freedom has made AM an ideal fabrication method for upper extremity splints.

Palousek et $\mathrm{al}^{14}$ reported an attempt to manufacture a wrist splint using AM technologies. The authors digitised the patient's forearm using a stereovision scanner, and modelled the splint with Solidworks CAD software (Dassault Systèmes Solidworks Corporation, 175 Wyman Street, Waltham, MA). Then, based on the digitised geometry, the splint was built using a Fused Deposition 
Modelling (FDM) machine using ABS plastic. Some tensile tests were carried out with ABS samples to compare the Ultimate Tensile Strength (UTS) against some traditional orthotic materials. However, this study did not attempt to fully exploit the design possibilities of AM and merely replicated an existing "off the shelf" splint design. For example, there was no attempt to introduce light-weight I ventilated lattice-type structures, multiple materials were not considered and traditional fasteners such as Velcro and buckles were retained. Furthermore, although an attempt was made to investigate the quality of fit, there was no simulation or experiment to investigate the physical performance of the AM splint compared to the mass-manufacture design. A more fundamental design for AM approach was reported in Paterson ${ }^{11}$ who proposed a customised 3D CAD strategy for splinting practitioners to design custom-made wrist splints in order to evaluate novel features only available through AM. This included the possibility of open, lattice-type structures that aimed to reduce weight, provide aesthetic patterns, and maintain a dry splint interior by increasing the ventilation subsequently reducing the moisture trapped between skin and splint or within the splint itself. Furthermore, due to the increasing number of potentially suitable AM materials available, AM splints could be cleaned at elevated temperatures and could be cleaned in domestic dishwashers for example, as suggested by Fried ${ }^{15}$ (depending on the exact specification of the materials 
being able to withstand the ranges of temperature encountered during cleaning cycles, without noticeable deformations).

The exploration of 3D CAD for upper extremity splinting conducted by Paterson ${ }^{11}$ addressed a CAD approach to splint design and developed a CAD workflow that maintained or enhanced the clinical decision making of the existing workflow. This research resulted in the design and prototyping of a number of AM wrist splints including a design that exploited multiple materials capabilities of the Objet Connex process. Whilst that study proved the feasibility of the CAD and AM approach it did not address physical / mechanical properties of AM splint designs. In order to address that limitation, it was considered that FEA was an appropriate tool in order to predict how the AM splint could react to real world loads. FEA has been previously used to assist the design of other orthotic devices for human body regions such as thoraco-lumbo-sacral region ${ }^{16}$, knee ${ }^{17}$, ankle-foot ${ }^{18}$, foot ${ }^{19}$ and thumb ${ }^{20}$ orthoses.

Consequently, this study aimed to investigate, by performing FEA simulations, the viability of using AM techniques to manufacture wrist splints in order to improve upon the typical LTT splinting approach. To achieve this goal, the simulations for both typical LTT and AM splints were prepared in terms of constraints, loads and material properties. Following on from this, several static simulations were performed. Von Mises stress values and displacements were 
compared for the two wrist splint designs. Subsequently, the physical prototype was manufactured via Poly-jet AM technology by using an Objet Connex printer. Finally, the physical prototype was evaluated under static conditions and the results compared to the computational simulations.

\section{Methods}

\section{Geometric models}

The AM splint design was the result of a research project performed by Paterson et al. ${ }^{21}$. The splint was specifically designed to be built using Objet Connex systems, exploiting the Poly-jet Matrix technologies that enable simultaneous multiple material deposition (Figure 1a). This AM splint design consists of seven elements (Figure 1b). The bulk of the splint is made up of two stiffer regions/volumes (labelled Dorsal inner and Wrap Around inner) with five additional more flexible regions/volumes: all with a thickness of $3 \mathrm{~mm}$. This thickness was considered appropriate as a first approximation bearing in mind the superior stiffness of the AM material and the weaker strength of the new design geometry due to the pattern of holes modelled on the surface. It should be noted that the digital design process proposed incorporates thickness as a variable. Clearly the exact thickness required would be optimised for a given splint design and the exact specification of AM material used. This study is the 
first stage in obtaining the data needed to specify the range of thickness required for the Objet Connex materials proposed. The soft elements enabled donning and doffing by providing a flexible "hinge". The soft patch illustrated the ability to relieve pressure around specific areas of anatomy. The soft edges were only incorporated to provide comfort when donning, doffing and everyday wear and were not intended to be structural. For the purposes of this research, several modifications were made in order to simplify and subsequently shorten the time to perform FE analysis. This involved removing the soft edges from the analysis. To maintain the correct geometry of the overall splint the soft edges were combined with their neighbouring stiffer elements; "Thenar", "Wrap around outer" and "Wrap around inner" were joined resulting in a single component. The "Hinge" and the "Patch" remained flexible but were joined together into one element. Finally, the "Dorsal inner" and the "Dorsal outer" were combined into one element (Figure 1c). The volume of the AM splint was $39 \mathrm{~cm}^{3}$ (Creo volume tool was used which has a 95\% accuracy). 

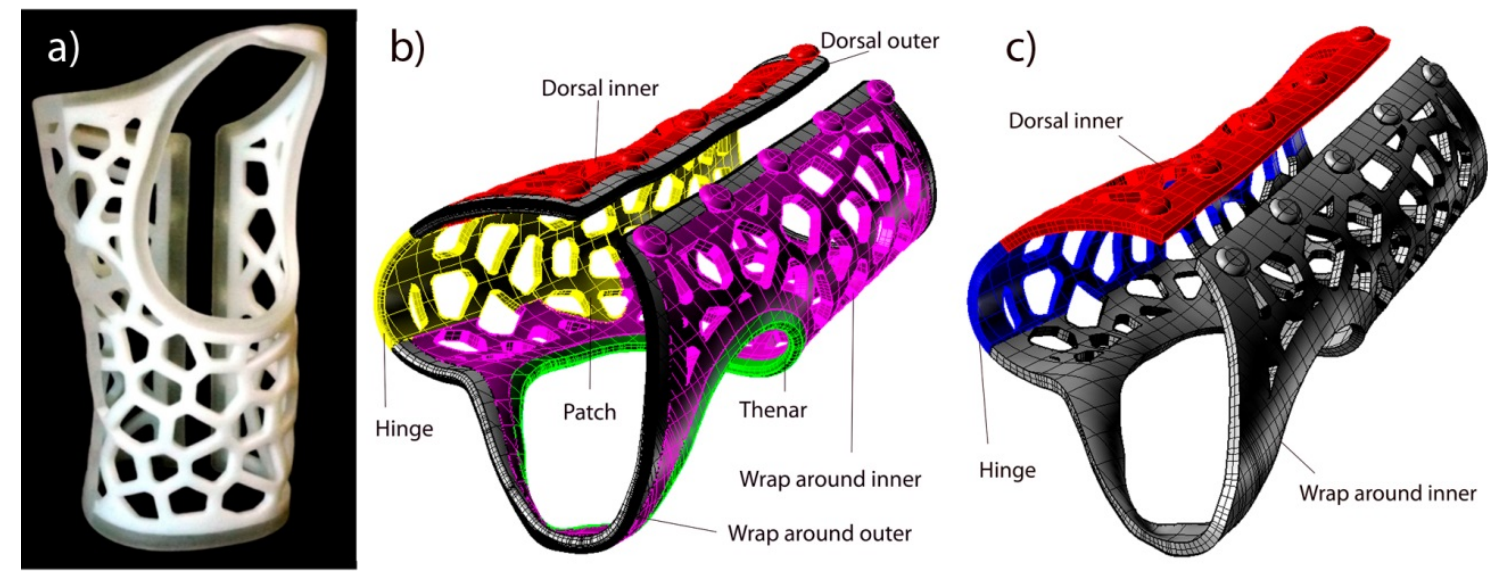

Figure 1. The AM splint 3D printed (a-left) and modelled (b-middle) by Paterson et al. ${ }^{21}$ and the simplified version used in this research (c-right).

A virtual model of a typical LTT splint was digitised from a patient-specific splint with an overall thickness of $2 \mathrm{~mm}$. This splint had been manufactured by an experienced splinting professional on a healthy volunteer. This volunteer was also used for the AM splint design enabling direct comparison of the two splint designs. Digitisation is often used for reverse engineering, where the topology of a physical artefact is transferred into a virtual representation by means of a suitable capturing device. The capturing device used in this instance was a ZScanner 800 3D laser scanner (3D Systems, Rock Hill, SC, USA); a noncontact hand held self-oriented scanner that consists of a laser emitter and three cameras. The laser light is projected onto the object's surface, and its reflection is captured by three cameras, which triangulate the laser within a 
specified focal area. According the manufacturer's specifications the highest resolution of the scanner is 50 microns while the accuracy is within 40 microns. The scanning procedure consisted of four steps:

1. Several reflective targets or 'markers' were randomly placed on or around the object surface

2. Using the acquisition software (Zscan V5.0 SR3), the user moved the scanner by hand around the splint to capture the locations of all of the markers

3. The laser power and the shutter speed of the scanner were manually calibrated using the acquisition software to capture white shiny surfaces

4. Once calibrated, following the same procedure as in the second step, the user moved the scanner around the splint in a freehand manner to capture the whole surface (provided at least four reflective markers were in view at any one time).

After scanning, the geometry was exported via the Stereolithography (STL) format and processed in Geomagic Studio 2013 (Geomagic, Morrisville, NC, USA) for geometric reconstruction. Automatic functions available in Geomagic were used to generate the final quilted NURBS model shown in Figure 2. The volume of the classic splint was $44 \mathrm{~cm}^{3}$. 

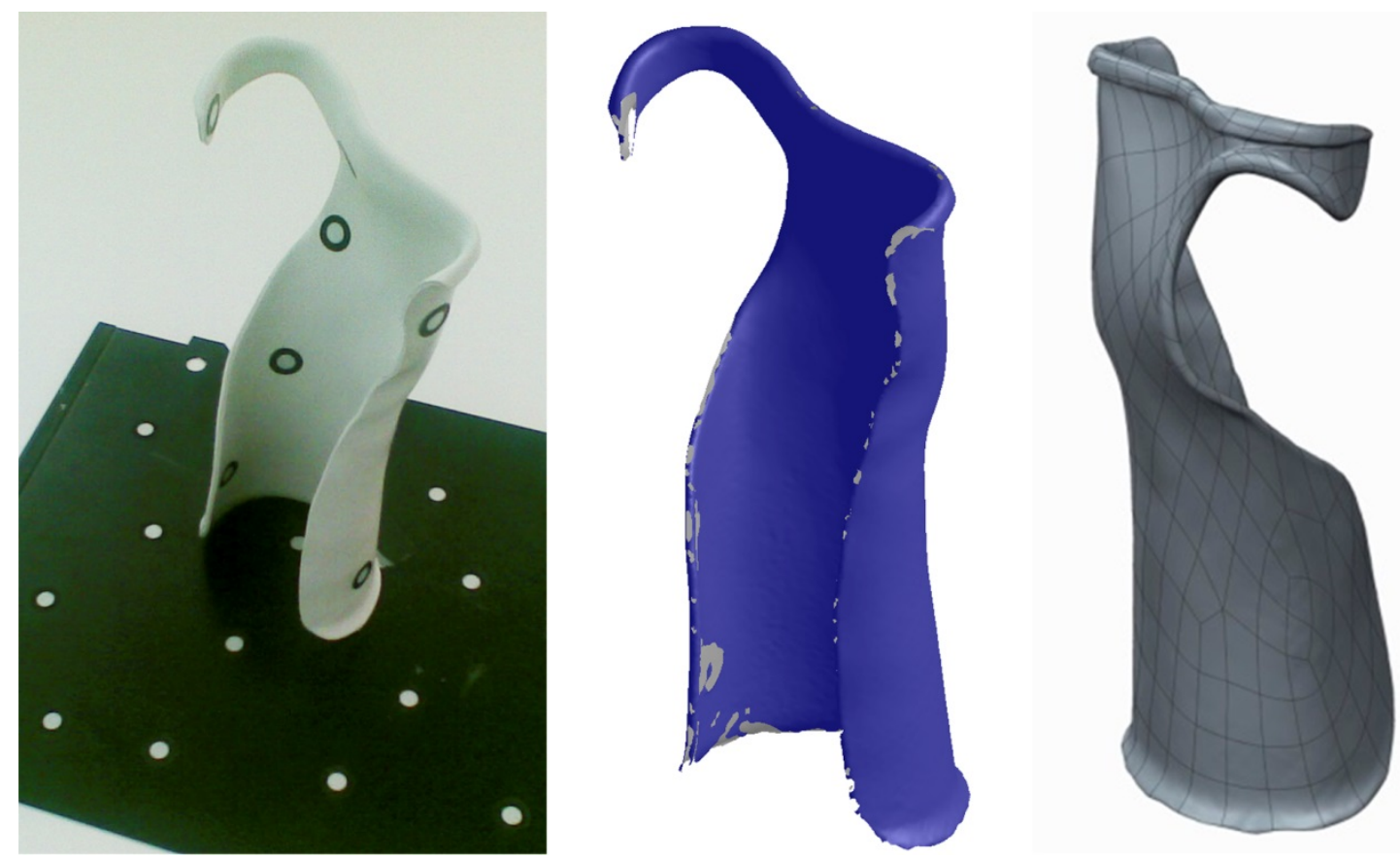

Figure 2. Reverse engineering process with the classic splint: reflective targets placed (left), geometry digitalised in ZScan (middle) and surface reconstruction with Geomagic Studio 2013 (right).

\section{Meshing}

The software used for the FE analysis was CREO 3.0 from PTC (PTC Corporate Headquarters, Needham, USA). Both the AM splint and the reverse engineered splint were imported into CREO via the Initial Graphics Exchange Specification (IGES) file format and then modelled using p-elements. The FE model was generated and resulted in 11,481 node points and 37,225 four node tetrahedral elements for the typical LTT splint and 16,851 node points and 37,148 four node tetrahedral elements for the AM splint (Figure 3). This 
meshing strategy using solid tetrahedrons was selected instead of a meshing strategy using shell elements. A shell element meshing strategy would have selected the internal (or external) surface of the splint and meshed it with shell elements at a constant thickness of $2 \mathrm{~mm}$. This strategy would have resulted in fewer elements and reduced simulation time. However, it was rejected due to the presence of the folded/rolled edges around the Thenar region and at the distal palmar crease. These two regions have a higher thickness and consequently have a higher stiffness. The use of constant thickness elements would not have replicated these rolled/folded regions and therefore would have influenced the displacement results undesirably. The axes were the same for both splints, that is, for a hand-wrist group in a pronated position, the $\mathrm{X}$ axis is positive radially (leftwards of the sagittal plane) and the $Z$ axis is positive downwards in a direction perpendicular to the palm (i.e. palm facing downwards). 

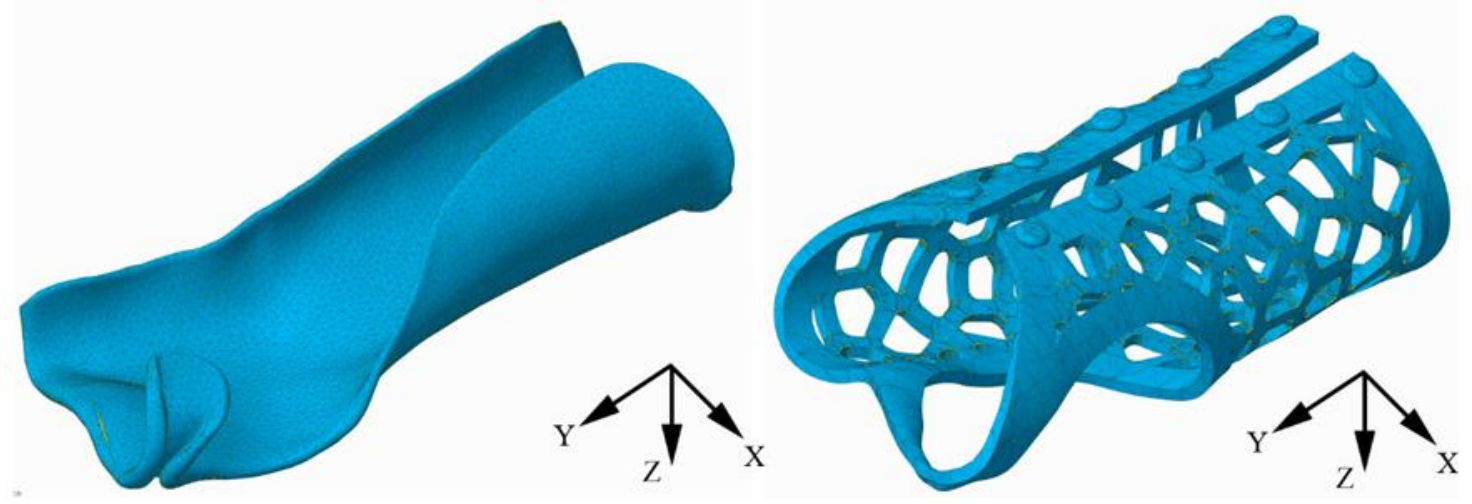

Figure 3: Mesh for the classic (left) and AM (right) splints.

\section{Materials}

For the AM splint, two different materials were selected: VeroWhitePlus (code name Fullcure 835) for the two stiffer regions/volumes and TangoBlackPlus (code name Fullcure 980) for the softer part. The range of Young's modulus for the stiffer material was obtained from Objet's data sheet ${ }^{22}$ (Stratasys Ltd, Minneapolis United States and Rehovot Israel). Hence, the minimum value was considered (2000 MPa). For the softer material, there was no information from the manufacturer regarding Young's modulus. The elastic modulus parameter was obtained from Ruiz de Galarreta et al $^{23}$, who investigated the manufacture abdominal aortas via 3D printing with the TangoBlackPlus material. They built tensile specimens using an Objet Eden 330 printer and according to ASTM D412 Type B. Tensile testing was performed on the specimens to generate force extension data using an INSTRON MINI 44 (Instron Worldwide, Norwood, 
MA) tensile test machine. The sample was subjected to a cross-head speed of $3.4 \mathrm{~mm} / \mathrm{min}$ until failure with a preconditioning of 10 cycles to $7.5 \%$ of the gauge length in order to avoid the Mullins effect ${ }^{24}$. This testing speed was selected in order to directly compare the testing results against data obtained from ex-vivo specimens available in the literature ${ }^{25}$. The load-displacement outputs from the tensile test machine were normalized to engineering stress-strain data. The resulting elastic modulus was $2.079 \mathrm{MPa}$. The Poisson's ratio was set to 0.34 for both materials ${ }^{26}$. The material properties were modelled as isotropic, which is a reasonable assumption despite differences in directional properties observed in some AM processes with the PolyJet technology. This assumption is also necessitated by the provision of only one value of elastic modulus supplied by the materials manufacturer that implies isotropic properties. The traditional splint was made from a Thermoplastic material called Polyflex (Patterson Medical Ltd, Huthwaite, UK). Its Young's modulus was obtained from tensile tests following the BS EN ISO 527-2:1996 standard. Ten different specimens, 5 in the horizontal direction and 5 vertically, were cut from a sample sheet using a Mercury II laser cutter (Grafityp UK Ltd, Tamworth, UK). The specimens were then tested using an INSTRON model 3366 tensile test machine with a $30 \mathrm{kN}$ load cell and fitted with a contact extensometer. Each sample was subjected to a cross-head speed of $1 \mathrm{~mm} / \mathrm{min}$. A support computer 
connected to the tensile machine was used to visualize the force-extension curves of each of the specimens and to obtain the Young Modulus for each specimen. Although the resulting engineering stress-strain graphs were nonlinear with decreasing stiffness as the load increased, only linear behaviour and small deformations were considered in the analyses.

The average and the standard deviation value of the ten specimens without including the lowest and the highest Young's values were considered. Hence, the average elastic modulus was $361 \mathrm{MPa}$ while the Standard Deviation was 39.1 MPa. The Poisson's ratio was obtained from literature for similar thermoplastics ${ }^{20}$ and set at 0.34 . The material properties were modelled as isotropic, although there could be some anisotropic properties in LTT splints due to temperature and stretching effects.

\section{Loads}

The motion of the wrist at the carpus level is mainly biaxial (2 Degrees Of Freedom) which results in four wrist movements: Flexion-Extension and RadialUlnar Deviation ${ }^{27,28}$. The wrist strength in each one of these movements is normally measured by means of volunteers subjected to static (isometric) and dynamic (isokinetic) tests. From the literature, two papers reported the maximum Flexion-Extension and Radial-Ulnar Deviation torques that healthy volunteers could perform ${ }^{29,30}$. However, these strength values should be taken 
with care when referring to wrists affected by arthritis. Amis et al. ${ }^{31}$ estimated that elbow flexion strength of rheumatoid patients was $45 \%$ of the normal range during Flexion-Extension movements in healthy patients. During real life wear scenarios, it is unlikely that patients with arthritis would willingly (nor have the capability in many instances to) achieve the maximum strength in both scenarios which could consequently break the splint. For this reason, in this research, the analyses were performed at $8 \%$ of the maximum isometric strength of a healthy person. Nevertheless, and to simulate the worst case scenario, the FE simulations were also run with a $50 \%$ of the maximum isometric strength of a healthy person by extrapolating these elbow strength conclusions to the wrist joint. Between both papers, the highest torques of Vanwearingen ${ }^{29}$ were chosen. The torque was divided by the distance between the metacarpophalangeal joints and the wrist to produce the input force $(\mathrm{N})$ necessary for the simulation ${ }^{32}$. A distance of $0.1 \mathrm{~m}$ was considered as representative for a $50^{\text {th }}$ percentile subject without any hand-wrist pathologies ${ }^{33}$. In order to avoid stress concentrations due to point forces, and to represent a more realistic interaction between the splints and the hand/wrist, the obtained force values were applied to the splint surfaces that are candidates to support those forces in real life (Figure 4 and Table 1). It is important to note that, aside from the most obvious surfaces under loads depending on each movement, the 
regions in contact with the thumb were also taken into account for the UInar movement of both splints whereas the palm region was considered only for the Radial movement of the typical splint.
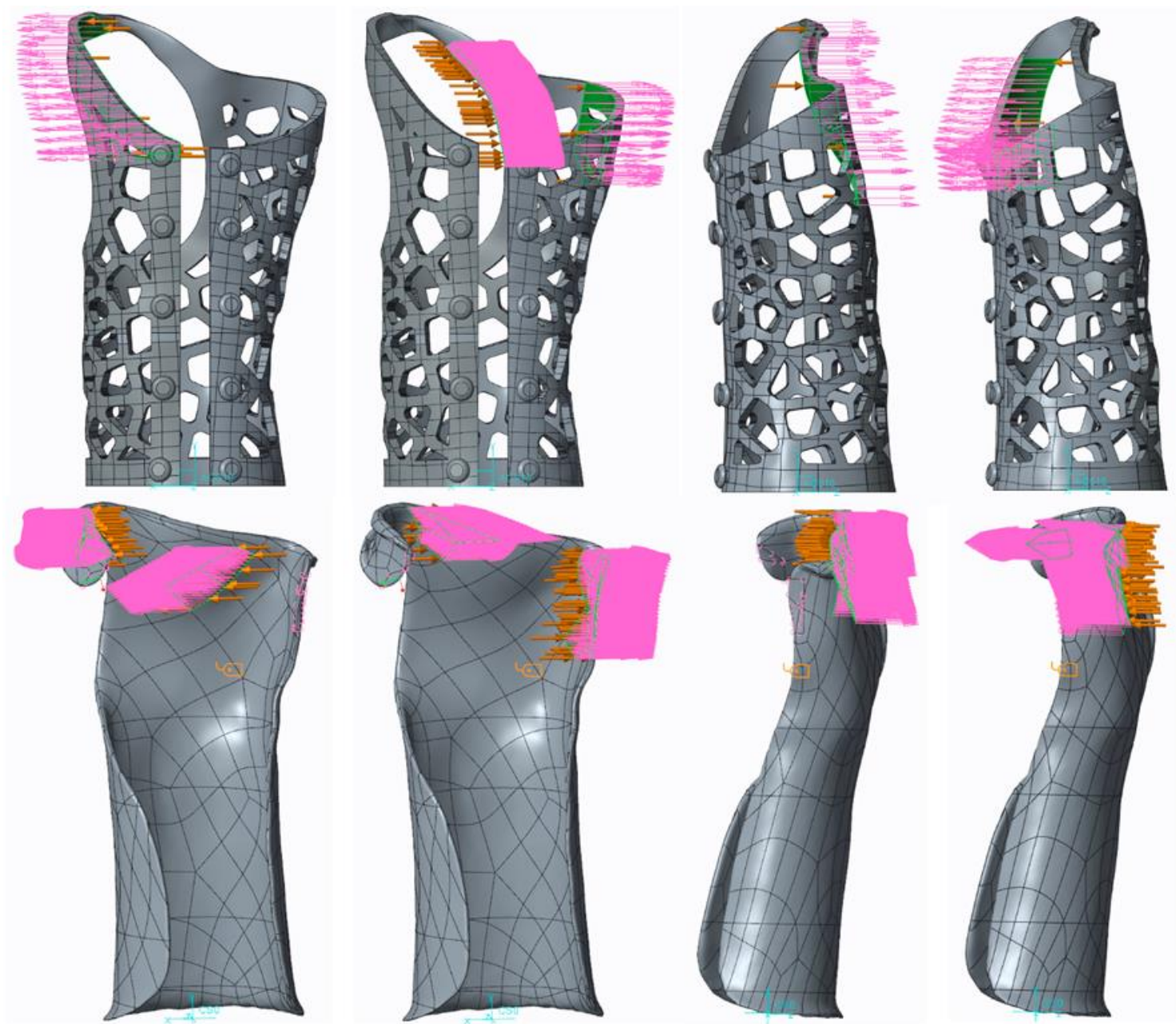

Figure 4. Load in the AM splint (top) and the classic splint (bottom) for the four main wrist movements. From left to right in this order: Radial, Ulnar, Flexion and Extension. 


\begin{tabular}{|l|l|l|l|l|}
\cline { 2 - 5 } \multicolumn{1}{|l|}{} & $\begin{array}{l}\text { Vanwearingen } \\
\text { Torque (Nm) }\end{array}$ & $8 \%$ Loads (N) & $50 \%$ Loads (N) & Applied \\
\hline Flexors & 14.8 & 11.9 & 73.7 & Z axis \\
\hline Extensors & 8.4 & 6.7 & 41.4 & -Z axis \\
\hline Radial deviators & 11.4 & 9.1 & 56.4 & X axis \\
\hline Ulnar deviators & 9.9 & 7.9 & 48.9 & $-X$ axis \\
\hline
\end{tabular}

Table 1. Considered torque and loads applied to the AM and classic splints for the 4 main movements.

\section{Constraints}

The typical splint is normally adjusted to the patient's arm by means of three Velcro strips to create a three-point pressure system ${ }^{6}$. Two of them are in the forearm and the third one is in the hand (see Figure 5). For the simulations, the patient's forearm was assumed to be at rest (or fixed) while the hand had free movement but limited by the splint itself. This way, the boundary condition was to rigidly join (3 translations and 3 rotations) the surfaces of the splint located at the Velcro sites in the forearm region. The third Velcro onto the hand was modelled as a rigid link: the surfaces, where the strip begins and ends at both sides of the sagittal plane of the splint, had no relative movement (Figure 5). 

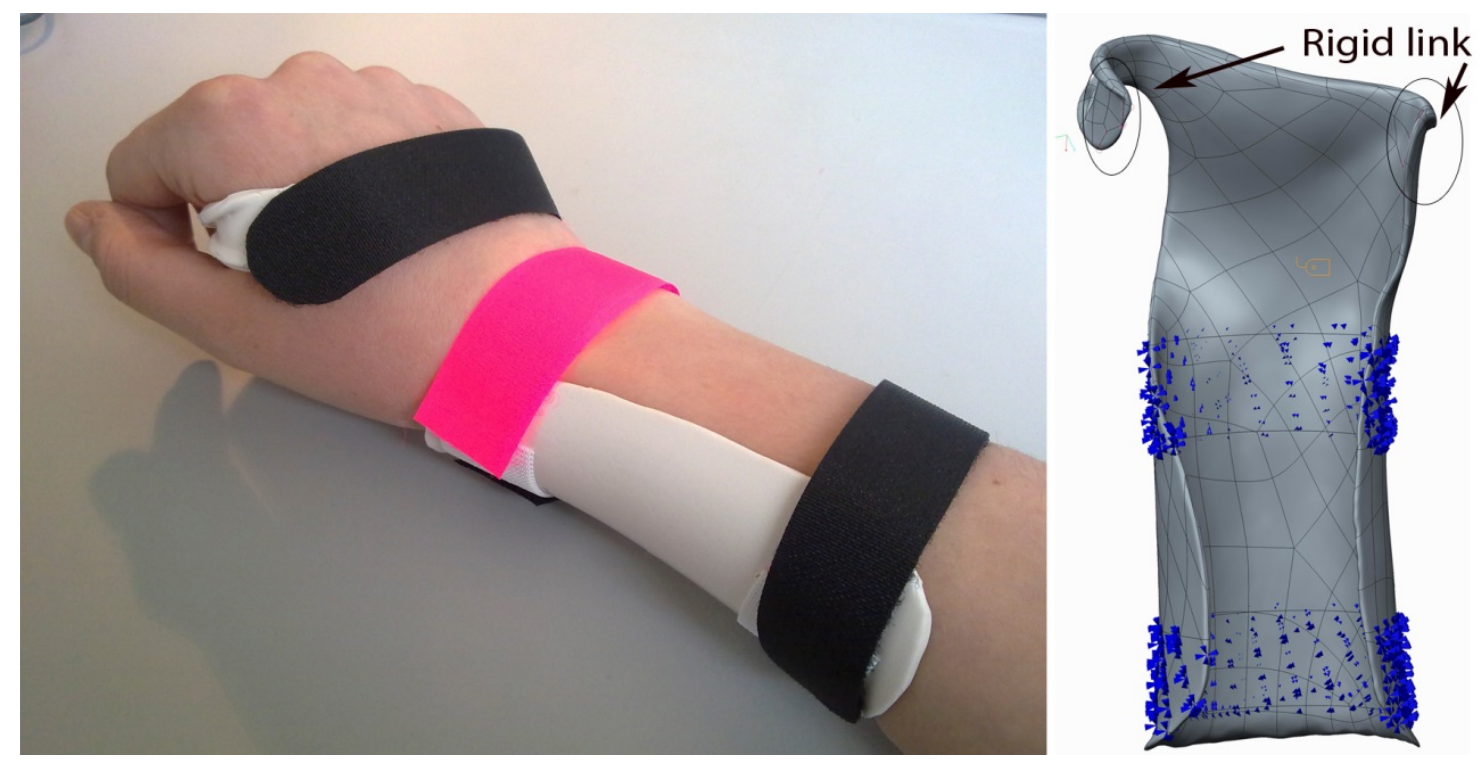

Figure 5. Velcro strips (left) and modelled constraints (right) for the classic splint.

In contrast to the typical splint, the AM splint is designed to wrap more around the hand and forearm and therefore donning and doffing is facilitated by enabling the splint to open and close with a flexible hinge. The splint is fastened using a rubber band which closes the splint in a manner similar to lacing a shoe. Similar to the traditional splint, the forearm was assumed to be at rest and the hand was free to move. Hence, the rear surfaces of the splint were fixed in 6 Degrees of Freedom (DOF) for the four movements. In addition, each movement had a second constraint on the middle surfaces of the splint to adjust the simulated forearm-splint interactions to the real ones creating the threepoint pressure system (Figure 6). 

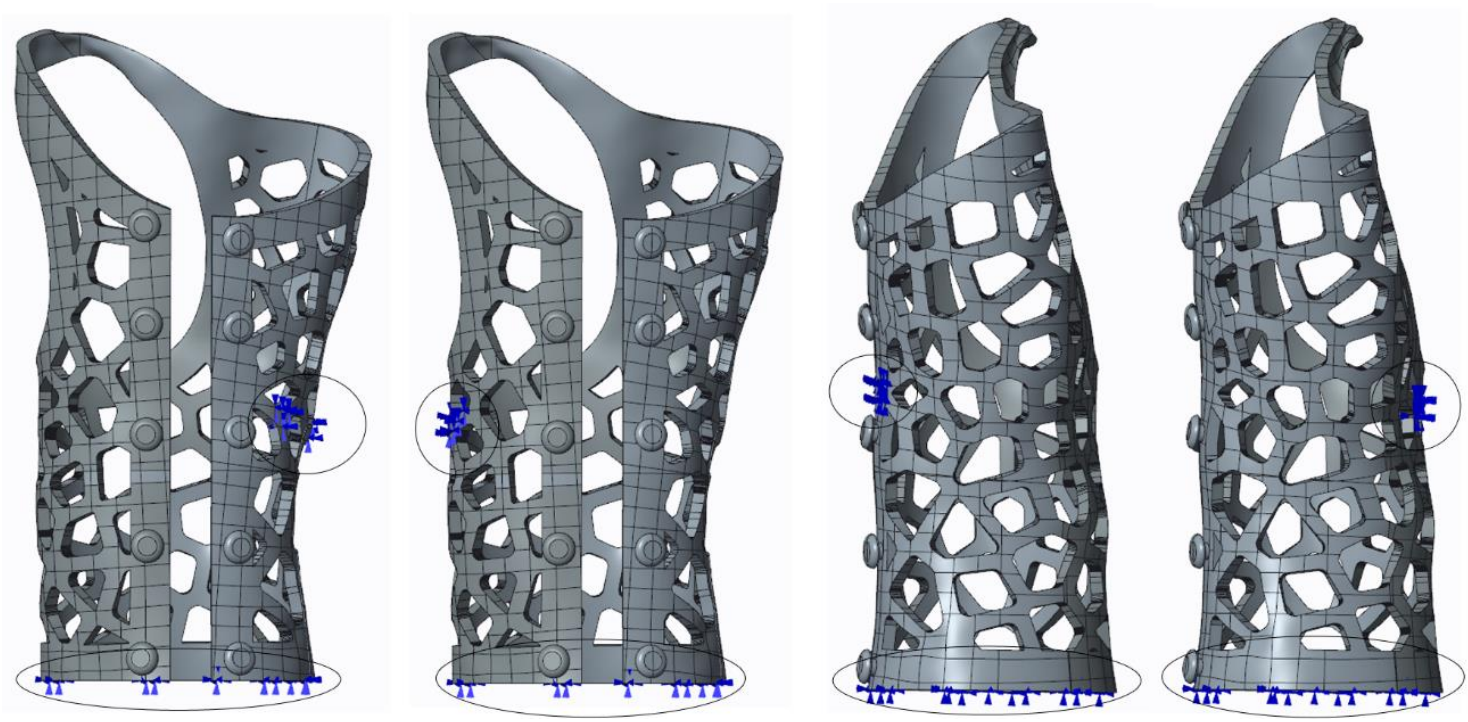

Figure 6. Constraints in the AM splint for the four main wrist movements. From left to right in this order: Radial, Ulnar, Flexion and Extension.

The rubber band was modelled as a series of spring idealisations with a linear stiffness of $6 \mathrm{~N} / \mathrm{m}$ obtained from simple weight tests. With the aim of comparing, the different alternatives to adjust the splint tightness with the rubber band, three options were considered and modelled with 2 (Option\#1), 6 (Option\#2) or 9 (Option\#3) springs. Several point forces $(0.4 \mathrm{~N}, 0.6 \mathrm{~N}$ and $0.78 \mathrm{~N}$ each) were applied to each spring for the 2, 6 and 9 spring configurations respectively, to model the preloads depending on the initial deformation of the rubber band (Figure 7). These loads were calculated by multiplying the initial deformation and the stiffness of the rubber band. The initial deformation in each configuration was calculated by subtracting the length of the rubber band once put in the splint from the length of the band without load. These deformations 
were 66,98 and $130 \mathrm{~mm}$. The stiffness of the rubber band was calculated with simple weight tests and resulted a figure of $6 \mathrm{~N} / \mathrm{m}$.

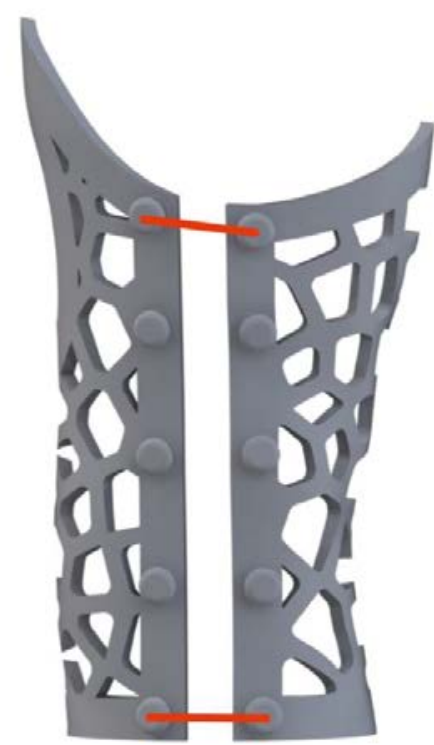

Option\#1

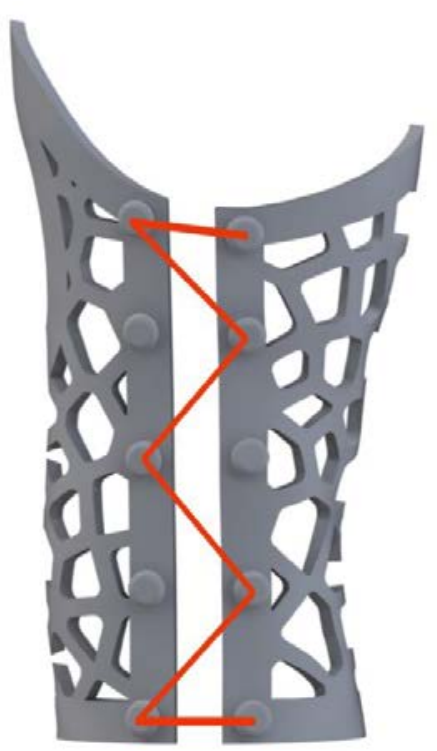

Option\#2

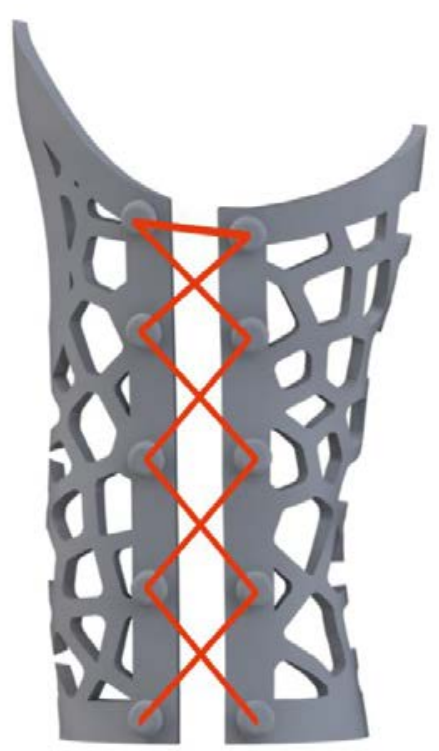

Option\#3

Figure 7. Spring configurations.

\section{Simulations}

Forty four simulations were performed in the four main movement directions: eight for the typical splint and thirty six for the AM splint. These simulations compared 1) the classic splint vs. the AM splint 2) the response of the AM splint depending on the rubber band configuration used for fastening and 3) the influence of the soft element (hinge) in the results of the AM splint. All 
simulations considered small deformations, meaning that the materials remained in their elastic region of the stress-strain curve. The solver method was the "Single-pass adaptive convergence method". This method runs a first pass at low polynomial order $(p=3)$ and determines a new $p$-order distribution to perform a second pass. The convergence control was also set as default, that is, the maximum stress error target in order to accept the simulation results as reasonable was $8 \%$. In our analyses we have observed that the RMS error estimate for stress in the classic model is lower than $1.2 \%$ while in the AM splint is below $0.7 \%$. The displacements and the Von Mises stress were obtained and compared. All simulations were run on a computer with a Core i7-4770 processor at $3.4 \mathrm{GHz}, 16 \mathrm{~GB}$ RAM and under Windows 7, 64 bit. Each simulation for both the traditional and the AM splint needed approximately 5 minutes.

\section{Physical testing}

A physical prototype of the new splint was created via AM for the physical testing. The AM splint was printed using the multiple material capabilities of an Objet Connex printer with Poly-jet technology. Connex printers use three different photopolymer materials for printing: two called 'model material' and the 'support material' which allows complicated geometries to be built. The printer used for this research project was the Objet Connex 500 (Stratasys Ltd., 
Minneapolis, MN, USA), which has a print resolution of 42, 42 and 30-microns in the $\mathrm{X}, \mathrm{Y}$ and $\mathrm{Z}$-axes respectively (when set up for multiple materials). The two 'model materials' used were VeroWhitePlus and TangoPlus, while the 'support material' was Fullcure 705 . The resulting prototype needed 16.12 hours, $150 \mathrm{~g}$ of VeroWhitePlus, $38 \mathrm{~g}$ of TangoPlus and $279 \mathrm{~g}$ of 'support material'. The 'support material' was then removed using hand tools.
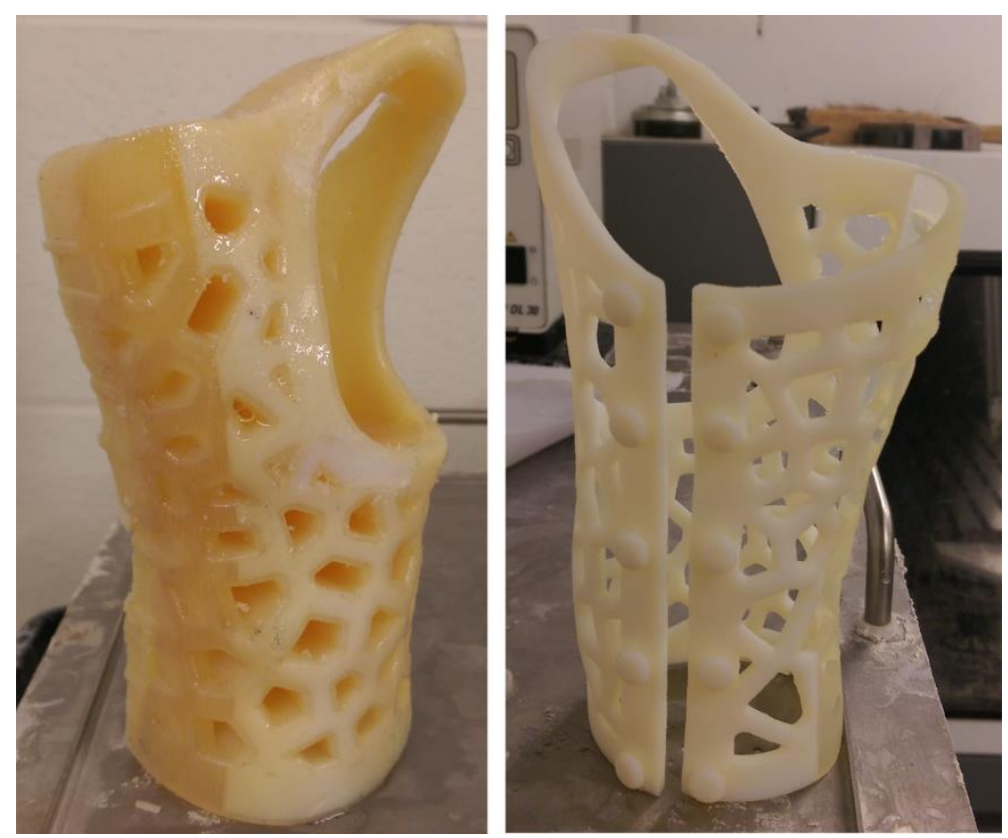

Figure 8. Physical prototype of the AM splint: As is after the printing process (left) and after postprocessing (right).

In order to explore the behaviour of the AM prototype during the four wrist movements, a series of static tests was performed using a tensile tester. A 
custom rig, loading and support modules were fabricated to allow the four movements (Ulnar, Radial, Flexion and Extension) to be tested (Figure 9).

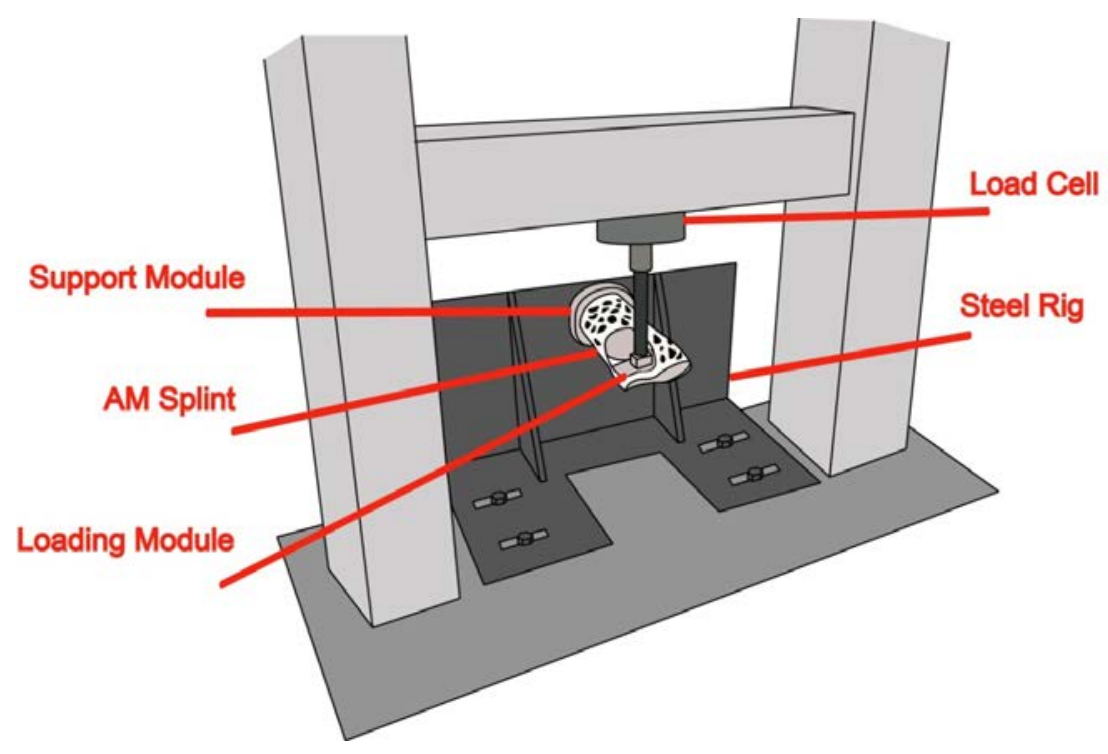

Figure 9. Physical testing set up.

The rig was constructed from steel $4 \mathrm{~mm}$ thick and consists of two plates at $90^{\circ}$ with two triangular gussets. Parts were cut using a Mach $3 \mathrm{~b}$ water jet cutter (Flow International, Washington, USA) and then welded together. The whole rig structure allows for any deflection seen confidently attributed to deflection in the splint only. The support module was designed using the hand scan data used to make the AM splint. This ensured a flush fit between the splint and the support module. Alignment tabs were built into the module to constrain the splint on the support module as can be seen in Figure 10. 

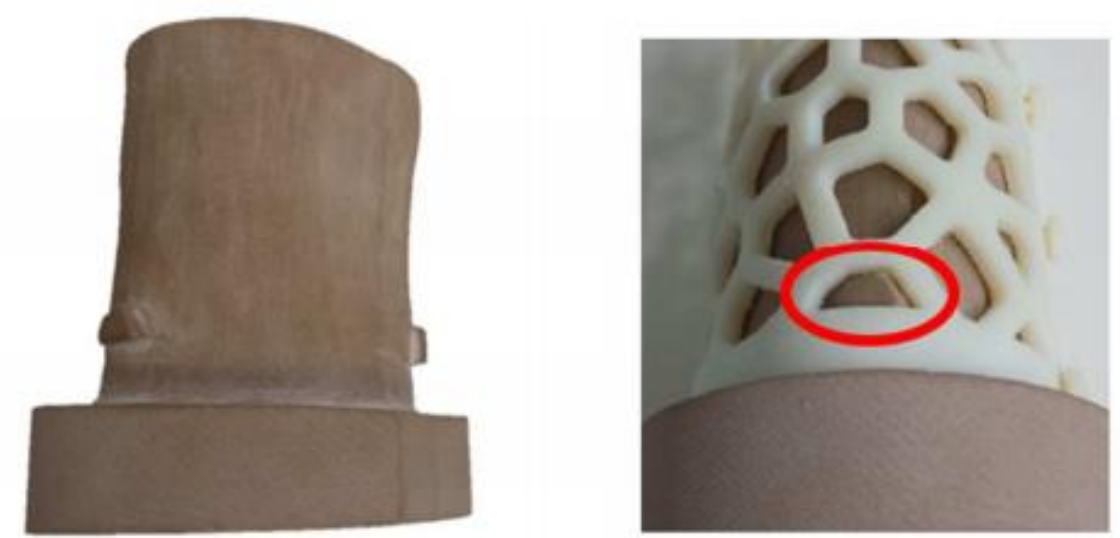

Figure 10. Support module with the alignment tabs (left) and the module with the AM splint (right).

A loading module was designed for each loading scenario. The surfaces that the loads were transmitted to on the splint were taken from the CAD model of the splint and are the same surfaces used in the FEA allowing for direct comparison. All loading modules were cut from EBA Board blocks using a CNC milling machine. Toolpaths were created in CREO 3.0. 


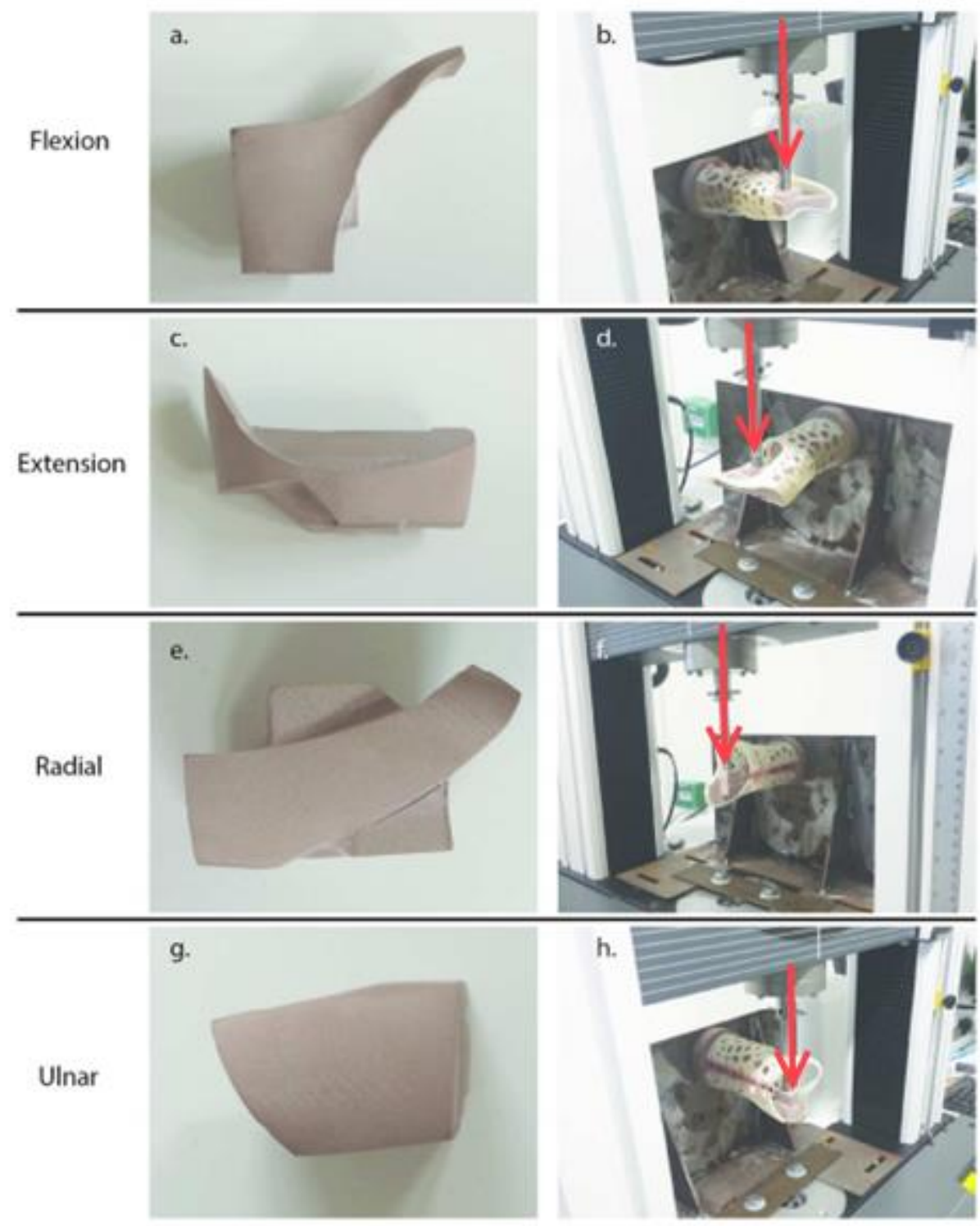

Figure 11. a,c, e and g: Loading Surfaces for respective load directions. Figure 11b, d, $f$ and $h$ : Load Test Set Up (Load lines shown in red). 
An Instron 3366 dual column tensile tester was used with a $5 \mathrm{KN}$ load cell. The splint was subjected to a cross-head speed of $4 \mathrm{~mm} / \mathrm{min}$ until the required load was reached. Each loading scenario was tested five times.

\section{Results}

A direct comparison between the AM and the traditional splint was carried out using the strongest lacing pattern for the elastic band (i.e. 9 springs Option \#3). The displacements obtained in the main axes from the FE simulations are presented in Table 2 for the $8 \%$ load while the absolute displacements and the Von Mises stress results are shown in Figure 12 through Figure 15.

\begin{tabular}{|l|l|l|l|l|l|l|}
\cline { 2 - 7 } \multicolumn{2}{c|}{} & \multicolumn{3}{|c|}{ Classic splint } & \multicolumn{2}{l|}{ AM splint } \\
\cline { 2 - 8 } & X & $Y$ & $Z$ & $X$ & $Y$ & $Z$ \\
\hline Flexors (Z) & 1.9 & 3.3 & 7.8 & 3.0 & 1.4 & 5.8 \\
\hline Extensors (-Z) & -1.2 & -1.9 & -4.6 & 0.6 & 0.2 & -1.1 \\
\hline Radial deviators (X) & 1.1 & -0.6 & 1.2 & 0.8 & -0.2 & -0.6 \\
\hline Ulnar deviators (-X) & -0.8 & 0.2 & -0.9 & -3.2 & -0.7 & 2.0 \\
\hline
\end{tabular}

Table 2. Maximum displacement in $\mathrm{mm}$ achieved in the main axes of both splints for $8 \%$ load. (Coupled axes with grey background). 


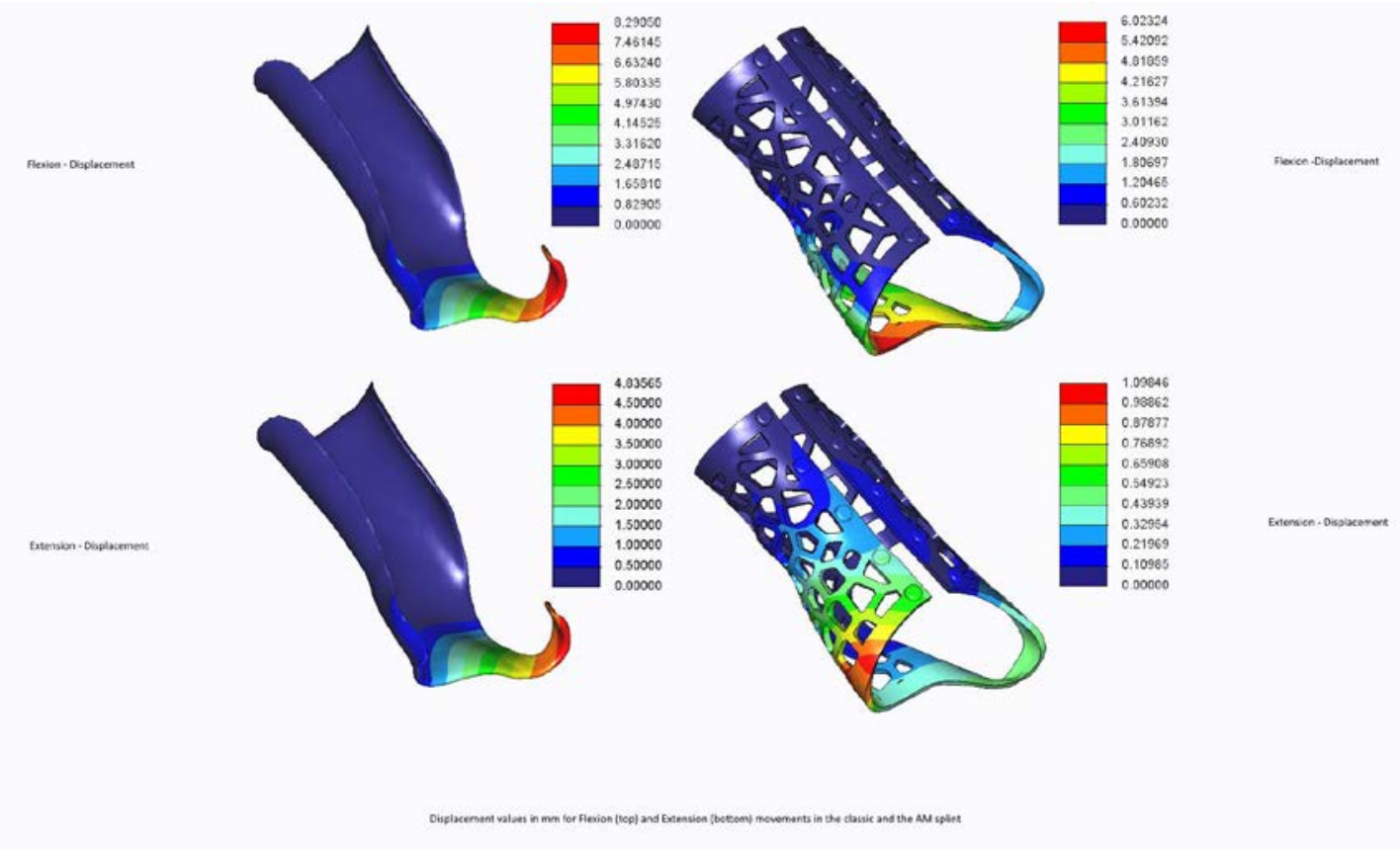

Figure 12. Displacement values in mm for Flexion (top) and Extension (bottom) movements in the classic and the AM splint. 

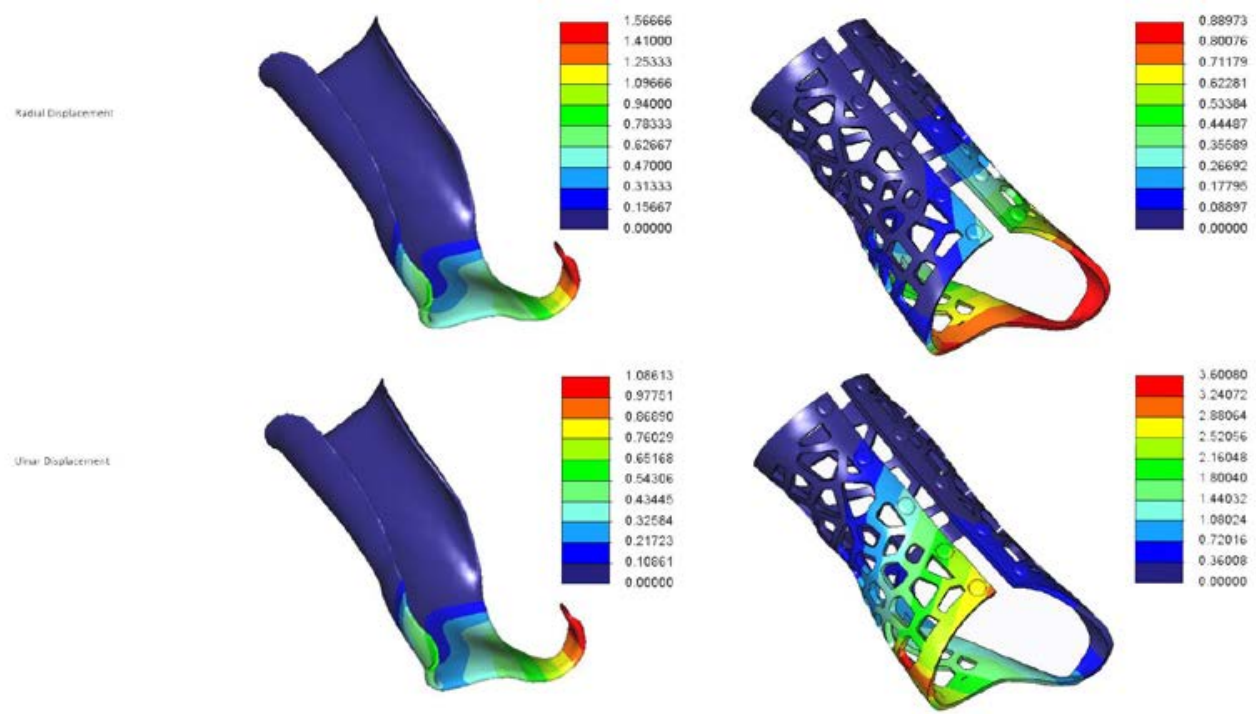

Figure 13. Displacement values in $\mathrm{mm}$ for Radial (top) and Ulnar (bottom) movements in the classic and the AM splint. 


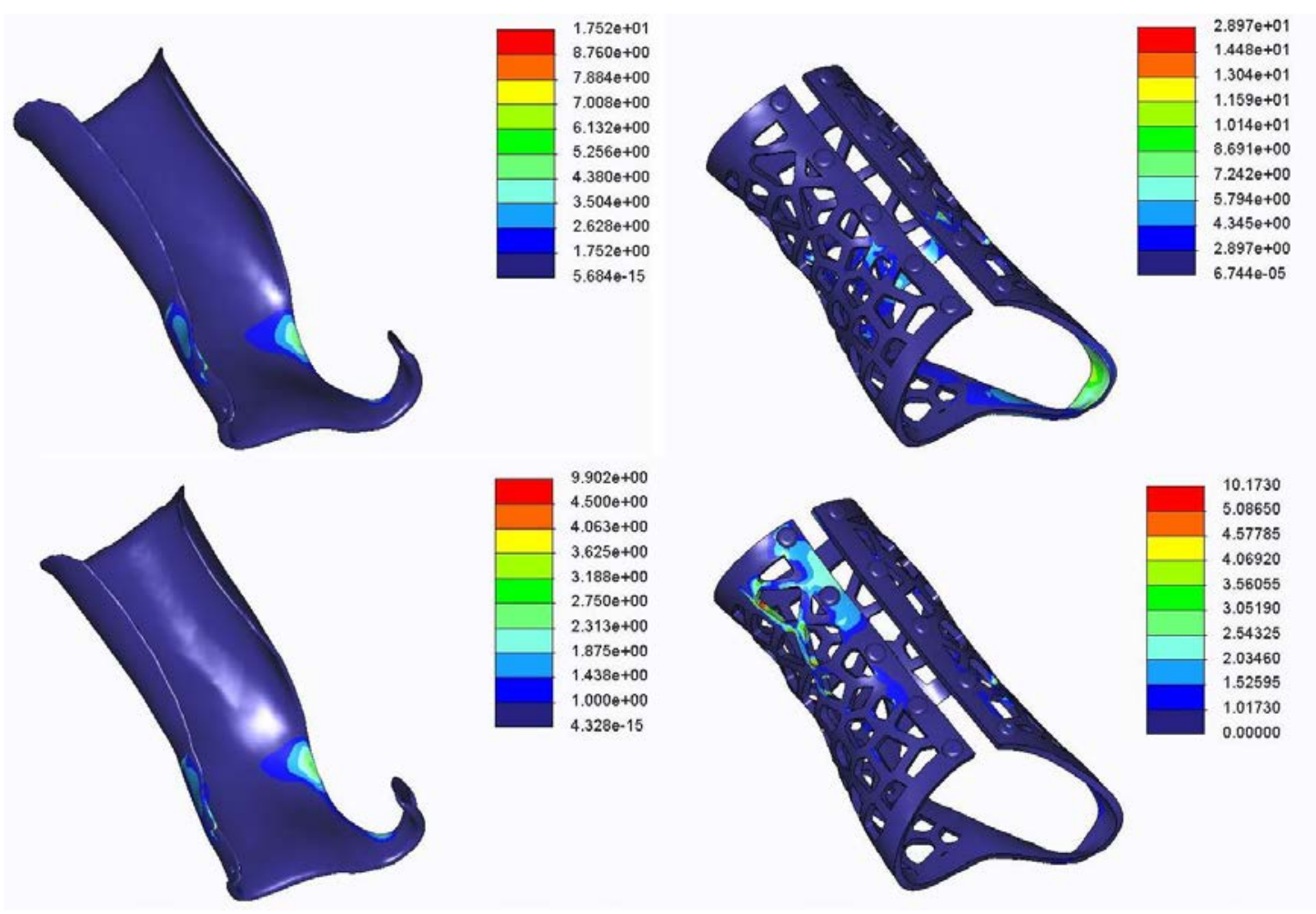

Figure 14. Von Mises stress values in MPa for Flexion (top) and Extension (bottom) movements in the classic and the AM splint. 

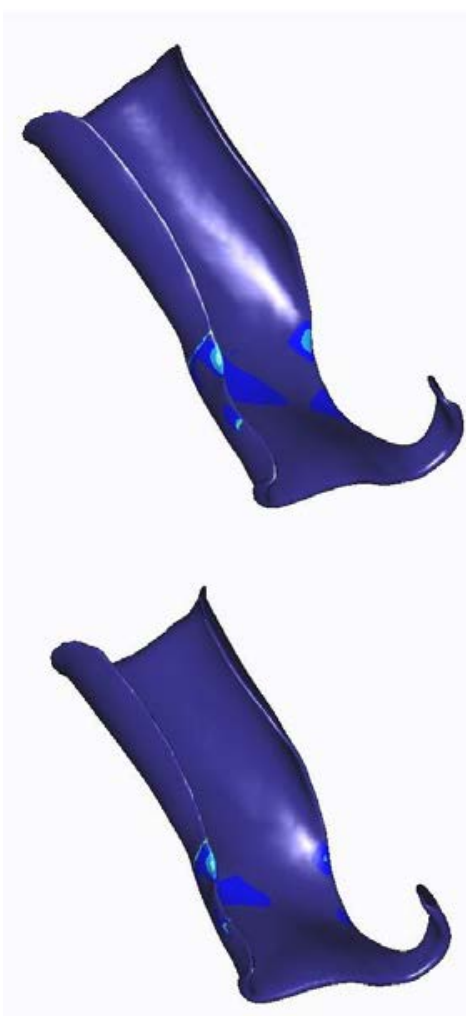
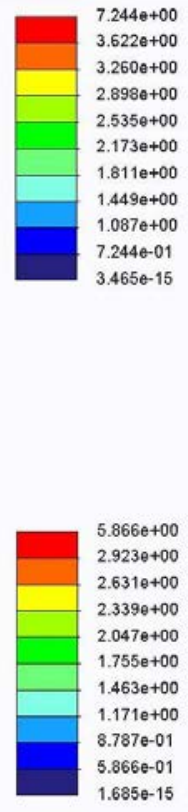

Figure 15. Von Mises stress values in MPa for Radial (top) and Ulnar (bottom) movements in the classic and the AM splint.

In terms of absolute displacements, the AM splint proved to be approximately $28 \%, 77 \%$ and $43 \%$ more rigid than the traditional splint in Flexion, Extension and Radial respectively. In the Ulnar directions the values were $70 \%$ lower in the traditional splint. Regardless of the loading direction was, the typical splint suffered the maximum displacement in the metacarpal bar. In the AM splint, there was no particular region that concentrated the maximum displacements independently of the loading directions. Taking a deeper look into the 
displacement in each axis, and especially to the coupled axes, this behaviour remained in Flexion and Extension movements, with a difference of 25\% and $76 \%$ respectively in favour of the AM splint. In the Radial direction, the displacements were almost identical: $0.8 \mathrm{~mm}$ and $1.1 \mathrm{~mm}$. However, this displacement reduction reverses in the Ulnar movement. Here, the maximum $X$ displacement is focused on the Hinge element of the AM splint and is $75 \%$ greater. This response was not unexpected after analysing the simulation, because some of the surfaces where the loads were applied belonged to the Hinge and it was modelled with very soft mechanical properties. Moving to the stress results, the classic splint shows stress peaks (17 MPa in Flexion) around the rigid link that modelled the hand Velcro strip. Depending on the type of LTT, they can exceed the tensile strength value (10-22 MPa) but without considering this region due to a stress concentration, the stresses were below $10 \mathrm{MPa}$. The other splint region that had notable stress values was the area of the splint closer to or in contact with the patient's wrist. They range from $2 \mathrm{MPa}$ to $6 \mathrm{MPa}$. In the AM splint, the surfaces close to the constraints had the highest values but were under the maximum tensile strength value (50-65Mpa according to Stratasys for the VeroWhitePlus), a value that, for polymers, coincides with the yield point and indicates the border between the elastic and the plastic behaviour. Just in case the splints were loaded with the full strength a 
rheumatoid wrist can perform (i.e. $50 \%$ load), the simulations, shown in Table 3 , indicate that the explained behaviour still remains. However, these last results are not completely valid and must be taken as an approximation of the splint deformation because the static analysis indicated that both splints exceeded their strength limits.

\begin{tabular}{|c|c|c|c|c|c|c|}
\hline & \multicolumn{3}{|c|}{ Classic splint } & \multicolumn{3}{|c|}{ AM splint } \\
\hline & $x$ & $\mathbf{Y}$ & z & $x$ & $\mathbf{Y}$ & $z$ \\
\hline Flexors (Z) & 11.7 & 20.7 & 48.4 & 20.5 & 7.1 & 36.7 \\
\hline Extensors (-Z) & -7.2 & -11.5 & -28.3 & -7.0 & 2.0 & -11.5 \\
\hline Radial deviators (X) & 7.0 & -3.6 & 7.5 & 6.4 & -1.8 & -6.0 \\
\hline Ulnar deviators $(-X)$ & -4.9 & 1.1 & -5.6 & -19.4 & -5.2 & -10.1 \\
\hline
\end{tabular}

Likewise, to check the influence in the displacements due to the rubber band fastener, the three possible options were analysed for $8 \%$ (Table 4) and $50 \%$ (Table 5) load. With the present constraints and loads, the rubber band configuration does not affect the displacement values. There were indeed changes in the displacements in the coupled directions, but they were negligible. The preload and the stiffness offered by the rubber band was so low 
in comparison to the applied loads, that they had almost no influence in the absolute displacement results. In general, the more the preload of the rubber band, the less the displacement of the splint. Although shown in the result table, the 'No Springs' option is redundant with the current constraints, because the AM splint itself would not work without any fastening system.

\begin{tabular}{|c|c|c|c|c|c|c|c|c|c|c|c|c|}
\hline & \multicolumn{3}{|c|}{ \#0 (No springs) } & \multicolumn{3}{|c|}{ \#1 (2 springs) } & \multicolumn{3}{|c|}{ \#2 (6 springs) } & \multicolumn{3}{|c|}{ \#3 (9 springs) } \\
\hline & $x$ & $\mathbf{Y}$ & Z & $x$ & $\mathbf{Y}$ & z & $x$ & $\mathbf{Y}$ & Z & $x$ & $\mathbf{Y}$ & $z$ \\
\hline Flexors (Z) & 2.9 & 1.4 & 5.8 & 2.9 & 1.4 & 5.8 & 2.9 & 1.4 & 5.8 & 3.0 & 1.4 & 5.8 \\
\hline Extensors (-Z) & -1.0 & -0.4 & -2.4 & -0.8 & -0.3 & -2.1 & 0.7 & 0.2 & -1.5 & 0.6 & 0.2 & -1.1 \\
\hline Radial deviators (X) & 1.2 & -0.4 & -0.9 & 1.1 & -0.3 & -0.8 & 1.0 & -0.3 & -0.7 & 0.8 & -0.2 & -0.6 \\
\hline Ulnar deviators $(-X)$ & $\mid-3.1$ & -0.9 & -1.8 & $|-3.1|$ & -0.8 & -1.7 & $\mid-3.1$ & -0.8 & -1.5 & -3.2 & -0.7 & 2.0 \\
\hline
\end{tabular}




\begin{tabular}{|c|c|c|c|c|c|c|c|c|c|c|c|c|}
\hline & \multicolumn{3}{|c|}{$\# 0$ (No springs) } & \multicolumn{3}{|c|}{ \#1 (2 springs) } & \multicolumn{3}{|c|}{ \#2 (6 springs) } & \multicolumn{3}{|c|}{ \#3 (9 springs) } \\
\hline & $x$ & $\mathbf{Y}$ & $z$ & $x$ & $\mathbf{Y}$ & $z$ & $x$ & $\mathbf{Y}$ & $\mathbf{Z}$ & $x$ & $\mathbf{Y}$ & Z \\
\hline Flexors (Z) & 20.5 & 7.1 & 36.6 & 20.5 & 7.1 & 36.6 & 20.5 & 7.1 & 36.7 & 20.5 & 7.1 & 36.7 \\
\hline Extensors (-Z) & -8.9 & 2.1 & $\mid-13.3$ & -8.5 & 2.1 & -13.0 & -7.9 & 2.0 & -12.4 & -7.0 & 2.0 & -11.5 \\
\hline Radial deviators (X) & 6.8 & -1.9 & -6.4 & 6.7 & -1.9 & -6.3 & 6.6 & -1.9 & -6.2 & 6.4 & -1.8 & -6.0 \\
\hline Ulnar deviators $(-X)$ & $\mid-19.7$ & -5.3 & -10.8 & -19.6 & -5.3 & -10.6 & -19.6 & -5.3 & -10.4 & -19.4 & -5.2 & -10.1 \\
\hline
\end{tabular}

On the other hand, and although the Hinge element is the key feature that allows proper donning and doffing for the patient, the new AM splint was also simulated to check how it performed in the absence of that rubber element. The TangoBlackPlus material was replaced with VeroWhitePlus. All results are shown in Table 6 , considering $8 \%$ load and once again only the rubber band of Option \#3. As expected, the coupled directions had less displacement for the rigid splint independent of the load direction. 


\begin{tabular}{|l|l|l|l|l|l|l|}
\cline { 2 - 8 } \multicolumn{1}{c|}{} & \multicolumn{4}{|c|}{ Fully rigid AM splint } & \multicolumn{2}{l|}{ AM splint } \\
\cline { 2 - 8 } & $\mathrm{X}$ & $\mathrm{Y}$ & $\mathrm{Z}$ & $\mathrm{X}$ & $\mathrm{Y}$ & $\mathrm{Z}$ \\
\hline Flexors (Z) & -0.5 & 0.4 & 1.3 & 3.0 & 1.4 & 5.8 \\
\hline Extensors (-Z) & 0.3 & -0.1 & -0.4 & 0.6 & 0.2 & -1.1 \\
\hline Radial deviators & & & & & & \\
\hline (X) & 0.5 & -0.2 & -0.2 & 0.8 & -0.2 & -0.6 \\
\hline Ulnar deviators (-X) & -0.3 & 0.1 & 0.5 & -3.2 & -0.7 & 2.0 \\
\hline
\end{tabular}

Table 6. Maximum displacement in $\mathrm{mm}$ in the AM splint if made fully or non-fully rigid for $8 \%$ load. (Coupled axes with grey background).

The experimental tests showed that the AM splint was capable of supporting the 8\% load of each loading scenario without failure as can be seen in Figure 16.

The comparison between the virtual and the physical displacements is shown in Table 7. 


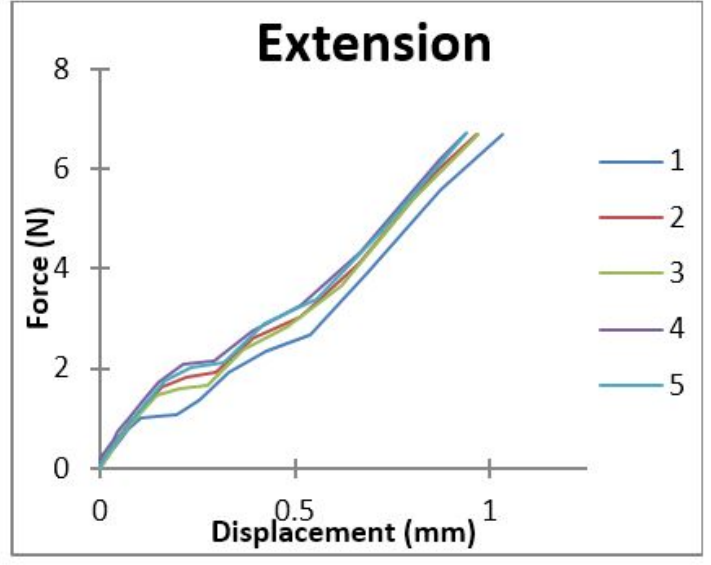

Figure 16 (a)

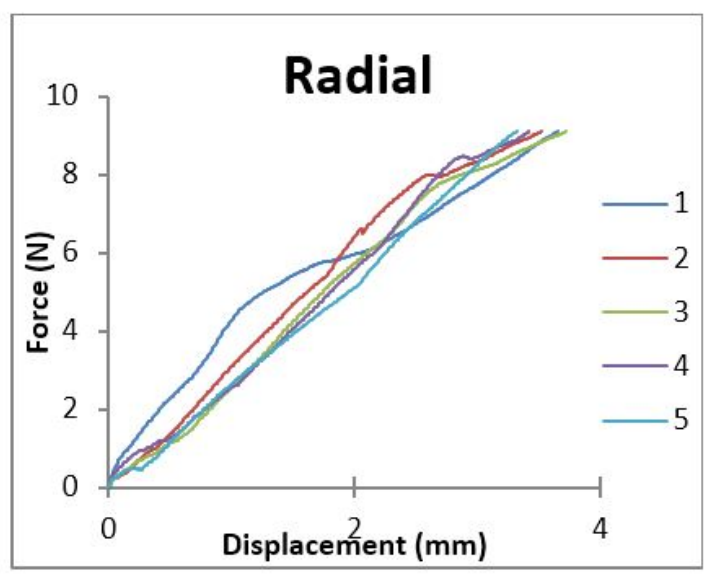

Figure 16 (c)

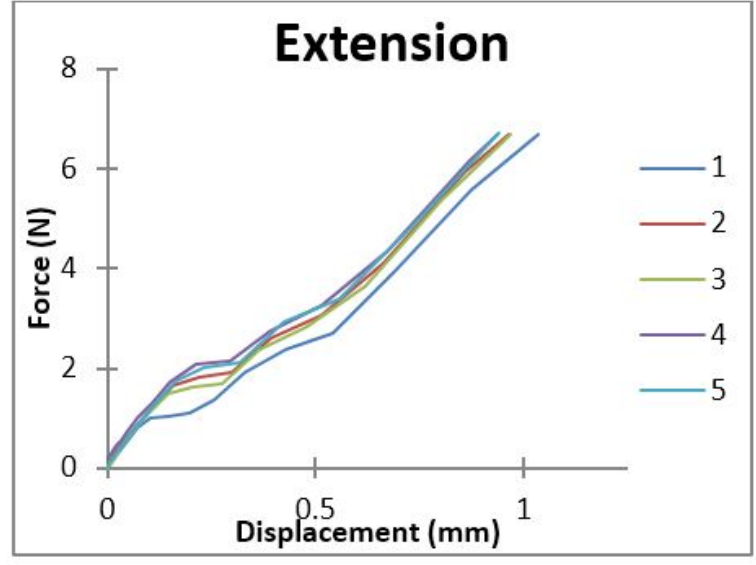

Figure 16 (b)

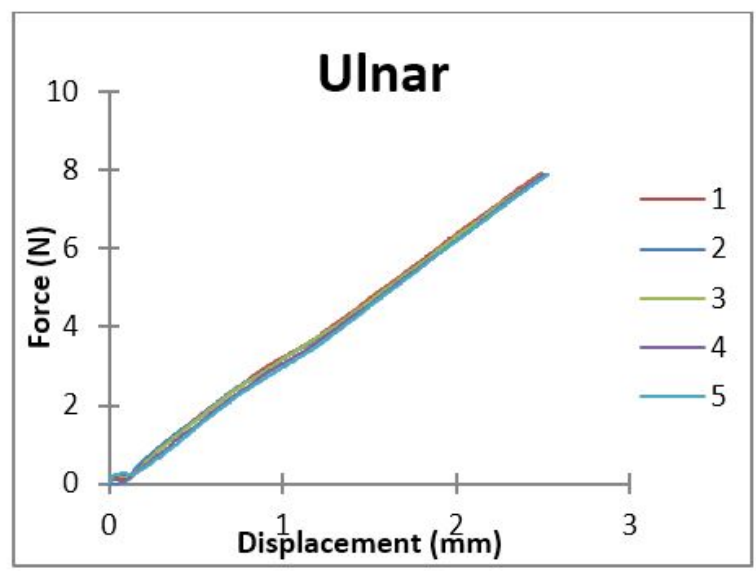

Figure 16 (d)

Figure 16. Experimental results for Flexion (a), Extension (b), Radial (c), and Ulnar (d) for AM splint. Lines refer to the number and sequential order of repeat tests carried out 


\begin{tabular}{|c|c|c|c|}
\hline & \multirow[b]{2}{*}{ Load (N) } & \multicolumn{2}{|c|}{ Displacement (mm) } \\
\hline & & Simulated & Actual \\
\hline Flexors & 11.90 & 6.02 & 3.46 \\
\hline Extensors & 6.60 & 1.10 & 0.97 \\
\hline Radial deviators & 9.10 & 0.89 & 3.53 \\
\hline Ulnar deviators & 7.90 & 3.24 & 2.51 \\
\hline
\end{tabular}

Table 7. Comparison of displacement between FEA simulations and physical testing for an $8 \%$ loading condition.

The Extension and Ulnar deviation showed a good correlation between the simulated and the experimental results, while the Flexion scenario showed a greater displacement during the virtual simulation. Physical displacement in the Radial direction was higher but remained at reasonable levels. After analysing simulations and experimental tests, it is possible to conclude that the rubber band fastening plays a bigger role in the stability of the splint.

A mathematical model was also completed to aid in the verification of the results of the traditional splint. This was done using Euler-Bernoulli beam theory. Euler Beam Theory is a simplification of linear theory of elasticity. The theory of elasticity states that when an applied stress is removed, the material 
returns to its un-deformed state ${ }^{34}$. A beam is a structural member subject to bending. Bending can occur in a beam when a load is applied perpendicular to the beam axis and at a distance from the support. A common type of beam support is a cantilever beam. This is when a beam is supported at one end and unsupported at the other ${ }^{35}$. Euler-Bernoulli beam theory can therefore be used to calculate the load carrying and deflection characteristic of a beam that is made of linear elastic material, where linear elastic materials are materials that deform proportionally to the applied load ${ }^{34}$. Using this definition it is possible to simplify a traditional splint being loaded by a patient to a cylindrical cantilever beam with a uniformly distributed load perpendicular to the beam's long axis. Using standard formulae for uniformly distributed loads on a cantilever beam, maximum deflection is found to be $3.81 \mathrm{~mm}$. When compared directly to the FEA results, of $4.6 \mathrm{~mm}$ for this loading configuration, it does not show much correlation. However, this FEA includes the thenar bar. When the FEA is analysed to obtain a displacement value negating the thenar bar, a closer correlation is found of $3.62 \mathrm{~mm}$ (Figure 17). Using stress/strain data gathered during the material properties testing mentioned previously, the displacement of 3.62mm can be correlated with an estimated stress of 6MPa for the LTT material. This value also correlates well with the FEA. 


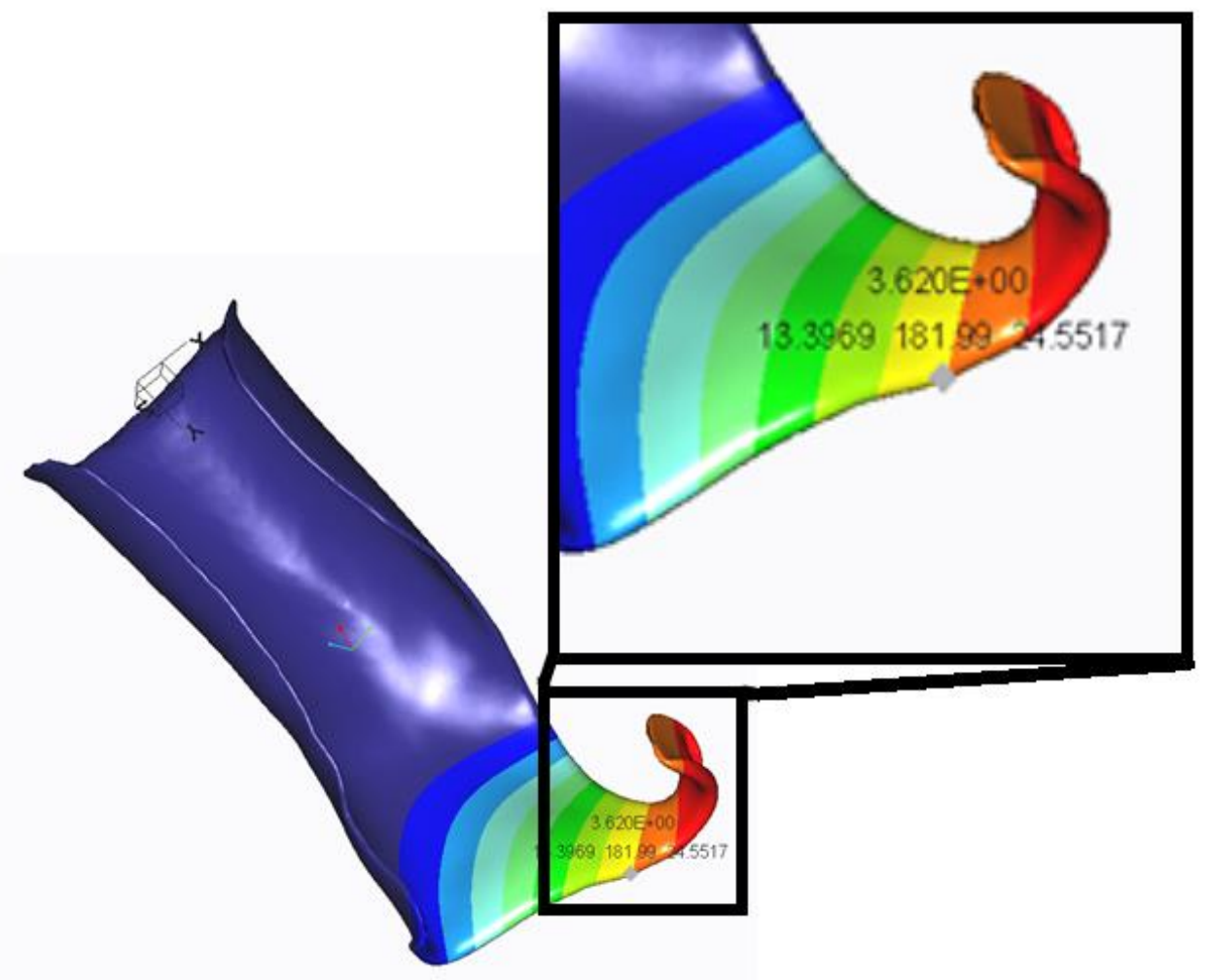

Figure 17: Deflection and XYZ coordinates of displacement ignoring thenar bar 


\section{Discussion}

The proposed constraints attempted to mimic the real life interaction between the patient's wrist-forearm group and their splint. However, there are some particular interactions that were simplified and not taken into account i.e. the relative movement between the forearm and the splint when the patient moves their hand, mainly because the Velcro strips and the rubber band do not perfectly fix the splint to the forearm.

The material properties also deserve a discussion as a limitation of this research. The mechanical properties used in the FE simulations came from two different sources: manufacturer datasheets and tensile testing. All these data were obtained for "ideal conditions" and may not reflect the real ones. In LTT, the tensile experiments were carried out with the original plastic sheet before all necessary stages involved in the splinting process. Throughout these stages, firstly, the LTT becomes flexible once it has reached the Glass Transition Temperature $(\mathrm{Tg})$ by placing it into a water bath at around $70^{\circ} \mathrm{C}$. And then before cooling, the practitioner needs to fit the splint to the patient's wrist by shaping the LTT sheet. In these two operations, the heat and the stretching could cause changes in the elastic modulus. For the AM materials the mechanical properties were applied without considering any anisotropic behaviour of the splint due to the printing process. This simplification was 
carried out considering that the manufacturer assumes an isotropic behaviour since they provide only a unique elastic modulus in the material datasheet. The effects of anisotropy will be investigated in future work. Additionally, this research does not take into account any reference to ageing of the material once printed. This aging could have direct consequences over time not only in terms of loss of dimensional accuracy, but also in the variation of the Young's Modulus as a result of exposure to wet and/or humid conditions, extremes of temperature or UV light, for example.

It is important to note that the results and conclusions of every FE simulation are completely dependent on the loads and the constraints applied. When deciding what the severity of the loads should be, no information was found within the literature regarding wrist joints with arthritis. Based on that lack of information and considering that the elbow and the wrist joint are physiologically similar, the authors assumed that the strength reduction in an arthritic wrist must be closer than in an arthritic elbow ${ }^{31}$. It would be interesting to incorporate the real forces that an arthritic patient would voluntarily apply to the splint in real life use.

Regarding the solver options and with respect to the mathematical equations in particular, the hypothesis of a linear stress-strain curve is valid for low loading conditions ( $8 \%$ for a healthy person). In our tensile experiments with the LTT, 
we noticed that the linear approximation could be considered excellent until 4 $\mathrm{MPa}$ and good enough from 4 to $6 \mathrm{MPa}$ (Figure 18). In the case of the AM material, according to the literature, the stress-strain curve behaviour is linear until $35-40 \mathrm{MPa}^{36}$. In our worst scenario (Flexion), the AM splint is below the 35-40 MPa limit while very few elements of the LTT splint are above $6 \mathrm{MPa}$, meaning that the linear approximation we made was reasonable.

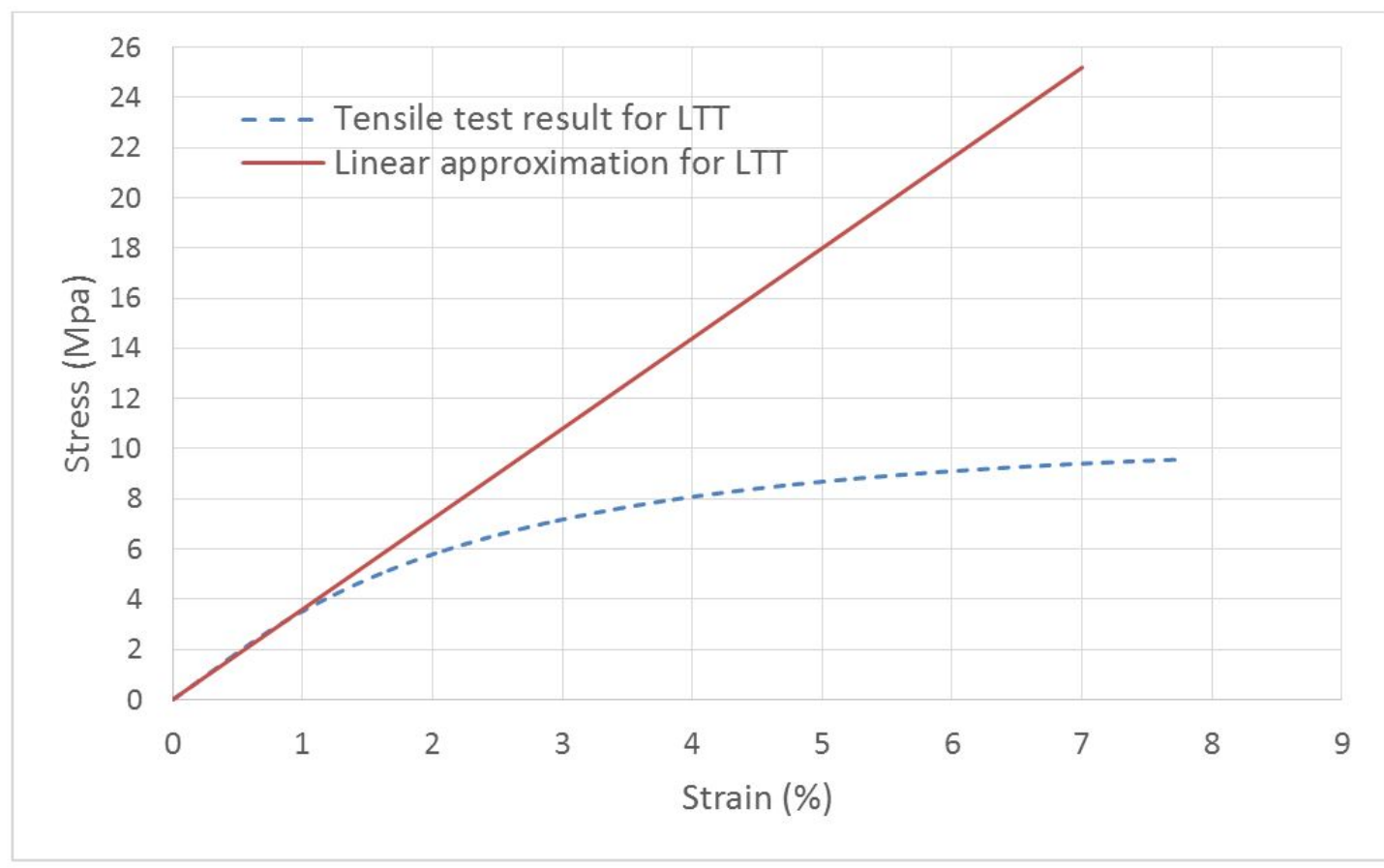

Figure 18: Engineering stress-strain curve for the LTT: linear approximation vs. tensile test

Nevertheless, the material non-linearity and the large deformation effects cannot be ignored for heavier loads (>8-9\%) meaning that more accurate simulations should be performed assuming larger deformations and the non- 
linear stress-strain curves from the tensile experiments of both the LTT and the AM materials. In the case of the LTT, the material could be modelled, for instance, either a) as a hyper elastic material whose equation for the strain energy potential followed a polynomial equation of order 2 , or, b) using a power law hardening curve in case an elastoplastic material is considered. This nonlinearity modelling will require much more computational time and would require a re-mesh of both splints in order to obtain accurate results within a reasonable time. However, we expect similar results for the $50 \%$ scenarios, since the real stress-strain curve is less stiff than the linear approximation and therefore the splint would behave even worse. As mentioned earlier, this should be carried out once the load that an arthritis patient can make to the wrist splint in real life use is known. Following this, either the small (linear) or the large (non-linear) deformation approach could be selected for future work. It would also be helpful to analyse more designs although this requires volunteers and considerable expense in design time, clinical time and materials. In the fullness of time, clinical trials will be needed to demonstrate the efficacy and cost-effectiveness of AM splints.

The current AM splint was designed to support low loading conditions with reasonable displacements (i.e. the splint is for immobilisation of an arthritic joint during rest and everyday tasks and it is not expected to be deliberately stressed 
to high level in normal use). However, when the loads were greater, the stresses experienced in the splint could, in some locations, approach or exceed the Ultimate Strength of the AM material (in this case 50-65 MPa for VeroWhitePlus). In order to ensure that any future AM splints do not risk physical failure due to localised stress exceeding the material's yield stress several different approaches could be taken. The first option would be to print the splint with a different AM material. For example, Stratasys supplies two materials with greater mechanical properties: an 'ABS-like' material whose minimum modulus of elasticity is $2600 \mathrm{MPa}$ and a high temperature material (code name RGD525) with an elastic modulus of at least $3200 \mathrm{MPa}$ according to the manufacturer datasheet. A second solution could be to increase the thickness of the whole splint uniformly, for example to a value greater than 3 $\mathrm{mm}$. However, this solution would imply a heavier splint and could consequently worsen the patient's handling in household tasks resulting in worse feedback compared to the traditional splint. A more sophisticated solution would be to redesign the splint in the specific regions where the stress is highest. This redesign could include designing splints with non-uniform thickness (i.e. locally thickening the structure) or changes in the lattice pattern (i.e. thicker struts where needed). In our digital design approach, the pattern can be restricted to specific areas and this could be applied to the locations with highest stress to 
keep them solid. When the AM splint was designed, it was intended to create a more breathable splint, among other benefits. This perforated design would not only need to be redesigned to strengthen the splint, but the higher contact forces from the smaller contact area indicative of a lattice design would also need to be investigated.

The final point of the discussion is the value of $A M$ for splint fabrication. AM technologies are becoming more and more popular amongst designers for building and then testing design prototypes (typically referred to as Rapid Prototyping) or even for building end-use products (Direct Manufacturing). Although these technologies are becoming more common and widely accepted by designers, it is true that adoption in many fields is still slow due to inherent limitations of the technologies, e.g. inadequate material properties, the limited number of materials available or high costs. However, the significant advantages of $A M$ will tend to minimise these drawbacks in future medical applications. From the design point of view, the AM approach allows the practice of user-centred design, enabling patient choice and input in terms of aesthetics (colour, pattern, etc.) in addition to custom-fitting and comfort. From an economic point of view, AM technologies are more affordable than several years ago and are likely to become more affordable in the future. Although the AM system used (Connex 500) in this research for the splint manufacturing is at 
the cutting edge of technology due to its multiple-material build capabilities, practitioners could obtain similar outputs from the less expensive 3D printers capable of manufacturing functional splints with comparatively little investment, saving money and manual labour time, although this would compromise the option for integral multiple materials. It would be necessary to conduct a thorough cost-benefit analysis for both approaches taking into consideration these different aspects (cost, learning curve for equipment use, etc.) and different working scenarios (a freelance practitioner against a practitioner employee of a public or private hospital). As mentioned previously the long-term properties of the materials needs to be investigated to ascertain adequate service life.

\section{Conclusions}

This study has documented that, from a technical point of view, the AM approach for the manufacturing of wrist splints has at least the same, or in certain respects an even better performance in terms of displacement, in comparison to the typical LTT approach. To this end, the authors have performed virtual simulations via FEA to compare the resulting stresses and displacements. The AM models for the simulations came from a previous research project while the LTT model was digitised from a real wrist splint using a non-contact scanner. The material properties for the classic splint were the 
result of tensile tests while the AM material properties were taken from the manufacturer supplied data sheet. The loads applied to the virtual splints were $8 \%$ of those applied by healthy people during experiments to measure the strength of the wrist joint. The results show that, in terms of absolute displacements, the AM splint was $28 \%, 77 \%$ and $43 \%$ more rigid than the classic splint in Flexion, Extension and Radial directions respectively. In the Ulnar direction, the AM splint presented greater displacement. In terms of Von Mises stresses, both splints remained under the tensile strength limits. The AM design was physically prototyped using a 3D printer and statically tested for the four main wrist movements, Flexors, Extensors, Radial Deviators and Ulnar Deviators, with maximum displacements of $3.46 \mathrm{~mm}, 0.97 \mathrm{~mm}, 3.53 \mathrm{~mm}$, and $2.51 \mathrm{~mm}$ respectively. These results reaffirmed the possibilities explored by other researchers and help to dispel the major concern of design engineers: the AM technologies are a real solution for wrist splint design because they are able to withstand the real life loads that a patient can make. The work demonstrates that AM splints are at least feasible and that further research and development is justified. 


\section{Funding}

The author(s) received no financial support for the research, authorship, and/or publication of this article.

\section{Acknowledgements}

Thanks to Ella Donnison, Patterson Medical Ltd. for the production of the LTT splint and provision of the LTT materials as well as other advice throughout this project. Thanks to Nigel Bunt and Sarah Drage from HK Rapid Prototyping Ltd. for the provision of the Objet Connex splint shown in
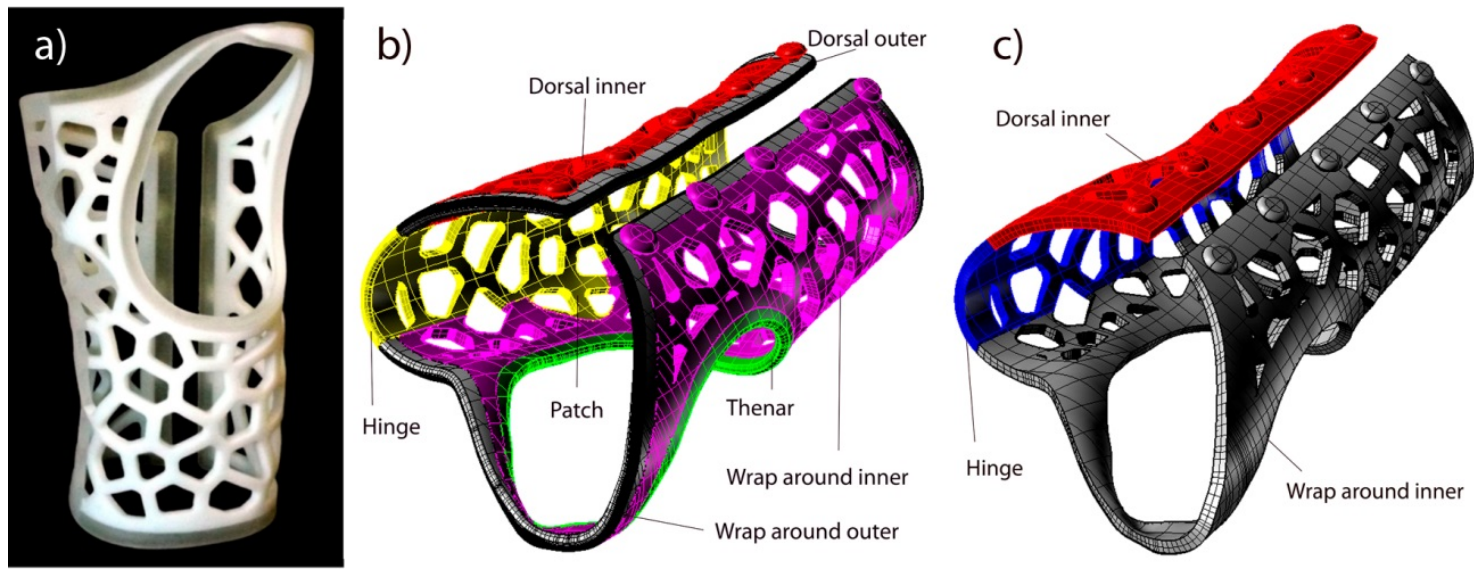

Figure 1.

Thanks to Andrew Sandaver from Wolfson School of Mechanical and Manufacturing Engineering for his help with the tensile tests of the LTT, Phil Dixon for his help with the production of the CNC parts, and Richard Myatt, for his help in constructing the test rig.

\section{Conflict of Interest}


The authors have no conflicts of interest. 


\section{References}

1. Landré-Beauvais AJ. The first description of rheumatoid arthritis. Unabridged text of the doctoral dissertation presented in 1800. Joint Bone Spine. 2001; 68: 130-43.

2. Borenstein DG, Silver G and Jenkins E. Approach to Initial Medical Treatment of Rheumatoid Arthritis. Archives of Family Medicine. 1993; 2: 54551.

3. Malahias M, Gardner H, Hindocha S, Juma A and Khan W. The Future of Rheumatoid Arthritis and Hand Surgery - Combining Evolutionary Pharmacology and Surgical Technique. The Open Orthopaedics Journal. 2012; 6: 88-94.

4. Radomski MV and Latham CAT. Occupational Therapy for Physical Dysfunction. 6th edition ed.: Lippincott Williams \& Wilkins, 2008.

5. Taylor E, Hanna J and Belcher HJCR. Splinting of the hand and wrist. Current Orthopaedics. 2003; 17: 465-74.

6. Coppard BM and Lohman H. Introduction to Splinting: A clinical reasoning \& problem-solving approach. Mosby, 2007.

7. Louise-Bender Pape T, Kim J and Weiner B. The shaping of individual meanings assigned to assistive technology: a review of personal factors. Disability and Rehabilitation. 2002; 24: 5-20.

8. Austin NM. Process of Splinting. Splinting the Hand and Upper Extremity: Principles and Process. Lippincott Williams \& Wilkins, 2003, p. 498.

9. Veehof MM, Taal E, Willems MJ and Van de Laar MA. Determinants of the use of wrist working splints in rheumatoid arthritis. Arthritis \& Rheumatism. 2008; 59: 531-6.

10. Coppanrd BM and Lynn P. Introduction to Splinting: A clinical reasoning \& problem-solving approach. Introduction to Splinting. St Louis, MO.: Mosby, Inc, 2001, p. 1-33.

11. Paterson AMJ. Digitisation of the splinting process: exploration and evaluation of a Computer Aided Design approach to support Additive Manufacture. PhD thesis at Loughborough Design School. Loughborough University, 2013, p. 259.

12. American Society for Testing and Materials International. ASTM F2792 12a Standard Terminology for Additive Manufacturing Technologies. 2012. 13. Gibson I, Rosen DW and Stucker B. Additive Manufacturing Technologies: Rapid Prototyping to Direct Digital Manufacturing. New York: Springer, 2010, p.462. 
14. Palousek D, Rosicky J, Koutny D, Stoklasek P and Navrat T. Pilot study of the wrist orthosis design process. Rapid Prototyping Journal. 2014; 20: 2732.

15. Fried S. Splint and or Method of Making Same. (2007) US Patent application 20070016323 A1 700/118; 602/5Edition G06F 19/00 (200601). 16. Liao Y-C, Feng C-K, Tsai M-W, Chen C-S, Cheng C-K and Ou Y-C. Shape Modification of the Boston Brace Using a Finite-Element Method with Topology Optimization. Spine. 2007; 32: 3014-9.

17. Stamenović D, Kojić M, Stojanović B and Hunter D. Pneumatic Osteoarthritis Knee Brace. Journal of Biomechanical Engineering. 2009; 131. 18. Syngellakis S, Arnold MA and Rassoulian H. Assessment of the nonlinear behaviour of plastic ankle orthoses by the finite element method.

Proceedings of the Institution of Mechanical Engineers, Part H. 2000; 214: 52739.

19. Cheung T-MJ and Zhang M. A 3-Dimensional Finite Element Model of the Human Foot and Ankle for Insole Design. Archives of Physical Medicine and Rehabilitation. 2005; 86: 353-8.

20. Huang T-H, Feng C-K, Gung Y-W, Tsai M-W, Chen C-S and Liu C-L. Optimization design of thumbspica splint using finite element method. Medical \& Biological Engineering \& Computing. 2006; 44: 1105-11.

21. Paterson A, Bibb R and Campbell R. Evaluation of a digitized splinting approach with multiple-material functionality using additive manufacturing technologies. 23rd Annual International Solid Freeform Fabrication Symposium - An Additive Manufacturing Conference. Austin, Texas, USA2012, p. 656-72. 22. Stratasys Ltd. Objet Materials Data Sheets. 2015.

23. Ruiz de Galarreta S, Cazón A, Antón A and Finnol E. Abdominal aortic aneurism: From clinical imaging to realistic replicas. Journal of Biomechanical Engineering Transaction of ASME. 2014; 136.

24. Mullins L. Softening of rubber by deformation. Rubber Chemistry and Technology. 1969; 42: 339-62.

25. Raghavan ML, Webster MW and Vorp DA. Ex Vivo Biomechanical Behaviour of Abdominal Aortic Aneurysm: Assessment Using a New Mathematical Model. Annals of Biomedical Engineering. 1996; 24: 573-82. 26. Ulu E, Korkmaz E, Yay K, Ozdoganlar OB and Kara LB. Enhancing the Structural Performance of Additively Manufactured Objects Through Build Orientation Optimization. ASME Journal of Mechanical Design. 2015; 137. 27. Youm Y, McMurthy RY, Flatt AE and Gillespie TE. Kinematics of the wrist. I. An experimental study of radial-ulnar deviation and flexion-extension. The Journal of Bone \& Joint Surgery. 1978; 60: 423-31. 
28. Volz RG, Lieb M and Benjamin J. Biomechanics of the wrist. Clinical Orthopaedics and Related Research. 1980; 149: 112-7.

29. Vanwearingen JM. Measuring Wrist Muscle Strength. The Journal of Orthopaedic and Sports Physical Therapy. 1983; 4: 217-28.

30. Delp SL, Grierson AE and Buchanan TS. Maximum isometric moments generated by the wrist in flexion extension and radial ulnar deviation. Journal of Blomechanics. 1996; 29: 1371-5.

31. Amis AA, Hughes $\mathrm{S}$, Miller JH, Wright $\mathrm{V}$ and Dowson D. Elbow joint forces in patients with rheumatoid arthritis. Rheumatology and Rehabilitation. 1979; 18: 230-4.

32. Glinsky J, Harvey L, Korten M, Drury C, Chee S and Gandevia SC. Short-term progressive resistance exercise may not be effective at increasing wrist strength in people with tetraplegia: a randomised controlled trial. Australian Journal of Physioteraphy. 2008; 54: 103-8.

33. Garrett JW. The Adult Human Hand: Some Anthropometric and Biomechanical Considerations. The Journal of the Human Factors and Ergonomics Society. 1971; 13: 117-31.

34. Rugarli P. Structural analysis with finite elements. Thomas Telford Ltd. ISBN: 978-0-7277-4093-9. 2010.

35. Seward D. Understanding Structures: Analysis, Materials, Design. Palgrave Macmillan. ISBN: 978-0230212633. 2009.

36. Pilipovic A, Raos $P$ and Sercer M. Experimental analysis of properties of materials for rapid prototyping. International Journal of Advanced Manufacturing Technology. 2009; 40: 105-15. 